Distribution Category UC-20c, d, \& e

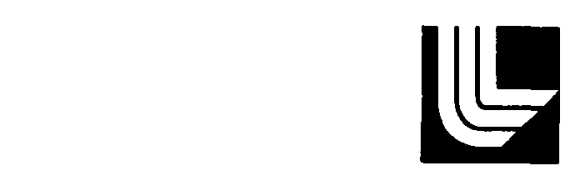

LAWRENCE LIVERMORE LABORATORY

University of California/Livermore, California/94550

\title{
UCRL-52811 \\ CATALYTIC OXIDATION OF TRITIUM IN AIR AT AMBIENT TEMPERATURE
}

\author{
A. E. Sherwood \\ B. G. Monahan \\ R. A. McWilliams \\ F. S. Uribe \\ C. M. Griffith
}

Manuscript date: July 1979

This document is PUBLICLY RELEASABLE 


\section{DISCLAIMER}

This report was prepared as an account of work sponsored by an agency of the United States Government. Neither the United States Government nor any agency Thereof, nor any of their employees, makes any warranty, express or implied, or assumes any legal liability or responsibility for the accuracy, completeness, or usefulness of any information, apparatus, product, or process disclosed, or represents that its use would not infringe privately owned rights. Reference herein to any specific commercial product, process, or service by trade name, trademark, manufacturer, or otherwise does not necessarily constitute or imply its endorsement, recommendation, or favoring by the United States Government or any agency thereof. The views and opinions of authors expressed herein do not necessarily state or reflect those of the United States Government or any agency thereof. 


\section{DISCLAIMER}

Portions of this document may be illegible in electronic image products. Images are produced from the best available original document. 
This wor $k$ was supported in part by Princeton Plasma Physics Laboratory under purchase order No. S-00817-K.

We wish to make the following acknowledgments:

W. L. Ross and L. D. Christensen contrived the original design concept for the maintenance enclosure.

C. A. Slettevold provided us with the catalyst pellet pore-volume and surface-area measurements. Electron-microprobe data were obtained by w. J. Steele. V. L. Duval carried out the chromatographic analysis.

R. Lyczkowski brought the hydrogen oxidation reference to our attention. G. A. Morris and V. P. Gede assisted us with many helpful discussions. F. W. Brunotts improved the manuscript with skillful editing. We are grateful to C. W. Pierce, Princeton Plasma Physics Laboratory, for his support throughout this project. 
Foreword

Symbols and Units

Abstract

Introduction

Experimental Apparatus

Enclosure Characteristics

Processing Loop

Sampling Loop

Ins trumentation

Tritium Gas-Pulse Injector

Catalyst Characterization

Pd/kaolin Catalyst

Pt/alumina Catalyst

$\mathrm{Pd} /$ zeolite Catalyst

Catalyst Physical properties

Electron Microprobe Scans.

Catalyst Activation .

Procedure and Method of Analysis

Catalyst Bed Fractional Conversion

Kinetic Rate Constant Determination

Experimental Test Data

Enclosure Drying Characteristics

$\mathrm{Pd} / \mathrm{kaolin}$ (unactivated)

$\mathrm{Pd} / \mathrm{kaolin}$ (age $=0.1 \mathrm{~d}$ )

$\mathrm{Pt} /$ alumina (age $=3 \mathrm{~d}$ )

Pt/alumina (age $=17 \mathrm{~d}$ )

$\mathrm{Pd} / \mathrm{kaolin}$ (age $=336 \mathrm{~d}$ )

Pt/alumina (age $=380 \mathrm{~d}$ )

$\mathrm{Pd} /$ zeolite (age $=1 \mathrm{~d}$ ).

Residual Tritiated Methane

$\mathrm{Pd} /$ zeolite $($ age $=35 \mathrm{~d}$ )

$\mathrm{Pd} / \mathrm{kaolin}$ (reactivated, age $=2 \mathrm{~d}$ ) 


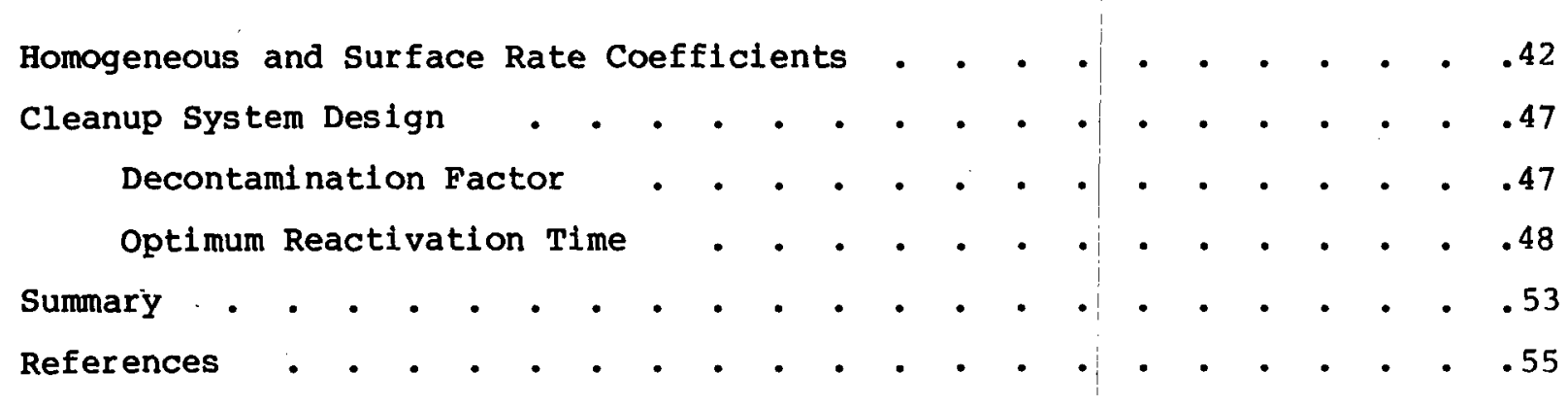




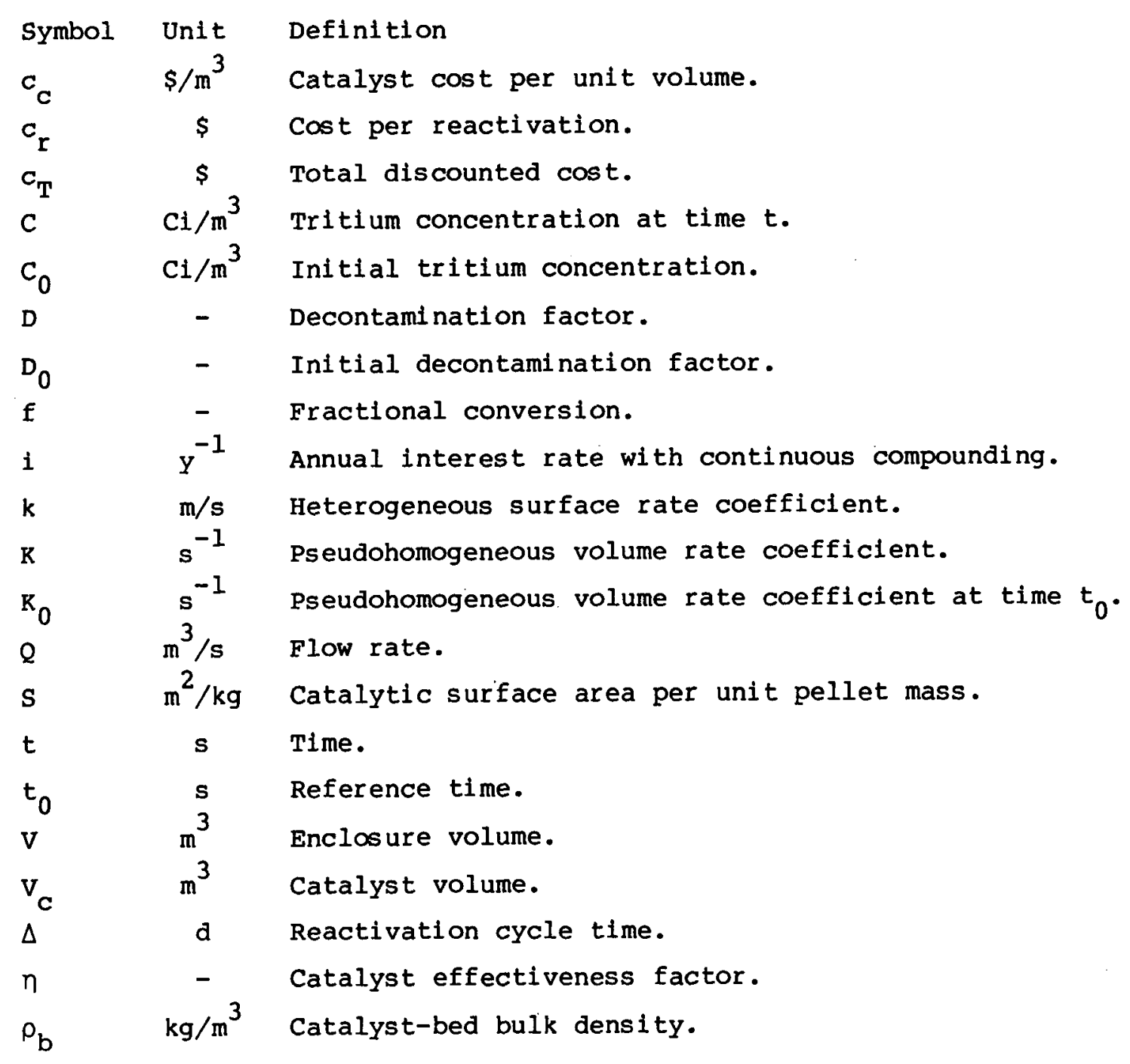




\section{CATALYTIC OXIDATION OF TRITIUM IN AIR}

AT AMBIENT TEMPERATURE

ABSTRACT

Tritium/air oxidation kinetic data are derived from ambient-temperature measurements carried out with three precious-metal catalysts. Each catalyst consists of a high-surface-area substrate in pelletized form, onto which precious metal has been dispersed. The metal/substrate combinations investigated are platinum/alumina, palladium/kaolin, and palladium/zeolite. Electronmicroprobe scans reveal that the dispersed metal is deposited in each case near the outer surface of the pellet, with metal concentration decreasing exponentially from the pellet surface. Kinetic oxidation measurements are made in a unique apparatus consisting of a large Lucite enclosure with an air atmosphere processed by a recirculating cleanup system. Dual-ionization chambers with an intermediate adsorption bed permit measurement of tritium gas and tritiated water vapor. Rate coefficients are determined from concentration decay following a spike injection of tritium into the enclosure. The catalytic reaction is first-order in tritium concentration in the range 10-10 $0^{5} \mathrm{\mu i} / \mathrm{m}^{3}$. Addition of hydrogen carrier gas is unnecessary. Each of the dispersed-metal catalysts is extremely active in promoting tritium oxidation in comparison with self-catalyzed atmospheric conversion; equivalent first-order rate constants are higher by roughly 9 orders of magnitude. The platinum-based catalyst is more effective than the palladium catalysts on a surface-area basis by about a factor of 3. Catalytic activity for all three catalysts declines with time of exposure to air after activation; following a power-law decay with an exponent of $-1 / 2$. A model for optimization of the catalyst reactivation cycle is proposed, revealing that the optimum catalyst volume scales with flow rate to the $2 / 3$ power. Ambient-temperature tritium oxidation is cost-effective for small- to intermediate-scale cleanup systems. A heated catalyst is desirable for large-scale systems. 
INTRODUCTION

Environmental releases from tritium-handling facilities can be minimized by passing tritium-contaminated waste gas or ventilation air through a scrubbing system. An effective two-step capture method is catalytic oxidation/ tritiated water adsorption. This method is the prime candidate for tritium containment technology on large-scale fusion experiments. 1, 2,3

There are no present competitors to catalysis/adisorption when oxygen or other chemically active species are present. Tritium may be recovered from inert gases by active-metal getter beds. ${ }^{4,5}$ some proponents of getter technology "view with alarm" the generation of tritiated-water waste by oxidation/ adsorption. We feel that the waste can be managed, and that the issue is simply cost-effectiveness. An effective oxygen-tolerant getter has yet to appear.

Process variations in the catalysis step are plentiful--for example, copperoxide catalysts at high temperatures and precious-metal catalysts at moderate to ambient temperatures. ${ }^{5,6}$ In a sense, tritium oxidation/adsorption is a mature technology. Yet it is more an art than a science. Only fragmentary performance information is available, which is of little use for the design of new systems. There is, in our opinion, a shocking absence of documented data on which to base system design. This situation prevails both for catalystkinetic data and for adsorbent-dynamics data.

This report presents tritium/oxidation kinetic data from experiments with three precious-metal catalysts. All experiments were carried out at room temperature--a regime that provides a severe test for catalyst effectiveness. The ability to operate at ambient temperature, without preheating and postcooling, is a great convenience to the system designer. This is especially true for standby emergency systems, and also for continuous operations.

We first describe the experimental apparatus, which was originally designed as a maintenance enclosure providing cleanup capability and safety during the disassembly and overhaul of tritium-contaminated equilpment. Measurements characterizing the catalysts are presented, followed by the experimental procedure and method of data analysis. Experimental test data are then described in detail, and the results summarized in terms of kinetic rate coefficients. Finally, we give some applications to cleanup system $\mid$ design based on an unexpected and striking feature of the kinetic results--the degradation with time of the rate coefficients. 
Experimental measurements were made using the apparatus shown schematically in Fig. 1, consisting of a large enclosure with associated processing and sampling loops.

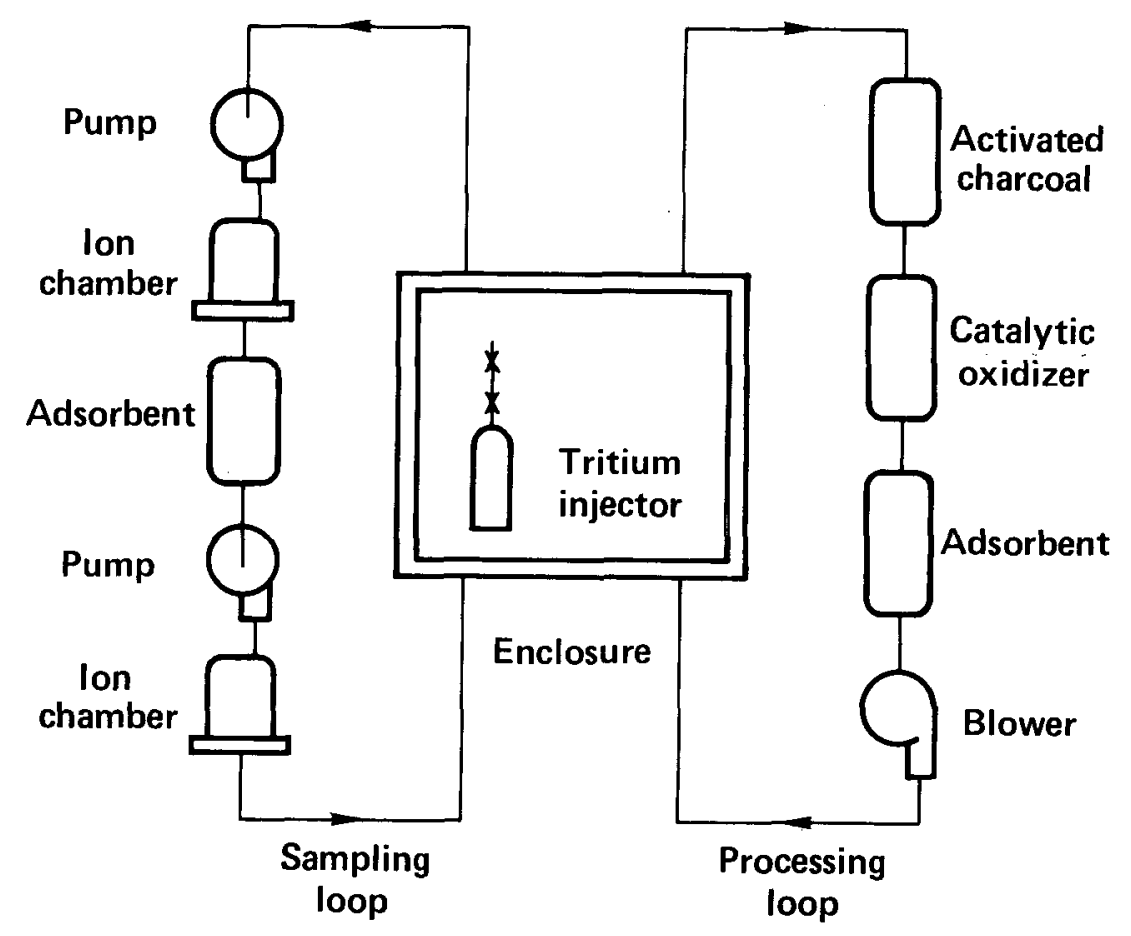

FIG. 1. Schematic diagram of experimental apparatus.

ENCLOSURE CHARACTERISTICS

The enclosure, shown in Fig. 2, is constructed primarily of Lucite (polymethylmethacrylate) supported by a steel framework. The door (1.1 $\mathrm{m}$ wide by $2.3 \mathrm{~m}$ high) permits access of large pieces of tritium-contaminated equipment for disassembly and maintenance. The enclosure is deslgned for ambient pressure operation. Neoprene gasket material is used to seal around the door and between the Lucite sheets and steel framework. No tritium leakage has been detected with modest pressure differentials up to about $1 \mathrm{kPa}$. The enclosure has an air-filled volume of about $5.45 \mathrm{~m}^{3}$. This volume includes an estimated 
$40 \ell$ of associated piping volume in the processing locp. Estimated enclosure surface areas are $10.5,5.9$, and $8.8 \mathrm{~m}^{2}$ for Lucite walls, Butyl gloves, and spray-painted metal surfaces, respectively.

PROCESSING LOOP

The processing loop and instrumentation are shown in Fig. 3. Tritiumcontaminated air is withdrawn from the enclosure through a cleanup system by a positive-displacement Roots-type blower, and then recilrculated back to the enclosure. Flow rate is adjustable, up to a maximum of about $10 \mathrm{~m}^{3} / \mathrm{h}$ with the present belt-drive and motor. Higher flow rates are possible, since the blower operates at a small fraction of rated $\mathrm{rpm}$.

Air flow passes in series through canisters containing activated charcoal particles, catalyst pellets, and zeolite adsorbent pellets. (See Fig. 1.) The purpose of the charcoal canister, which was not fillecl during our experiments, is to remove trace contaminants that might poison the catalyst. The catalyst bed promotes the oxidation of tritium gas to tritiated water, and the adsorbent bed removes tritiated water from the flowing air. Liride $5 \mathrm{~A}$ molecular seive zeolite pellets (nominal $1 / 8 \mathrm{in}$. diameter) are used for water adsorption.

A cutaway view of one of the processing loop canisters is shown in Fig. 4. A removable packed-bed cartridge $(9.74 \mathrm{~cm} i . d$.$) rests on a ledge within the$ canister. The canister and cartridge are constructed of 304 stainless steel. Air flow is downward through the loose-packed pellet bed. Porous, sintered steel filter discs (nominal $65 \mu \mathrm{m}$ pore size, 1/4 in. thickness) at each end of the cylindrical cartridge hold the pellets in place. Huntington MV-150 bellows-seal valves are used in the processing loop.

SAMPLING LOOP

A separate sampling loop draws air from the enclosure through two ionization chambers separated by an adsorbent bed, as shown in Fig. 1, and then recycles the air to the enclosure. Flow rate in the sampling loop is adjustable up to about $8 \mathrm{l} / \mathrm{min}$, which is ample to follow the relatively slow changes in tritium concentration within the enclosure. Metal Bellows Corp. MB-4l pumps are used for circulation in the sampling loop. The adsorbent bed is similar in 


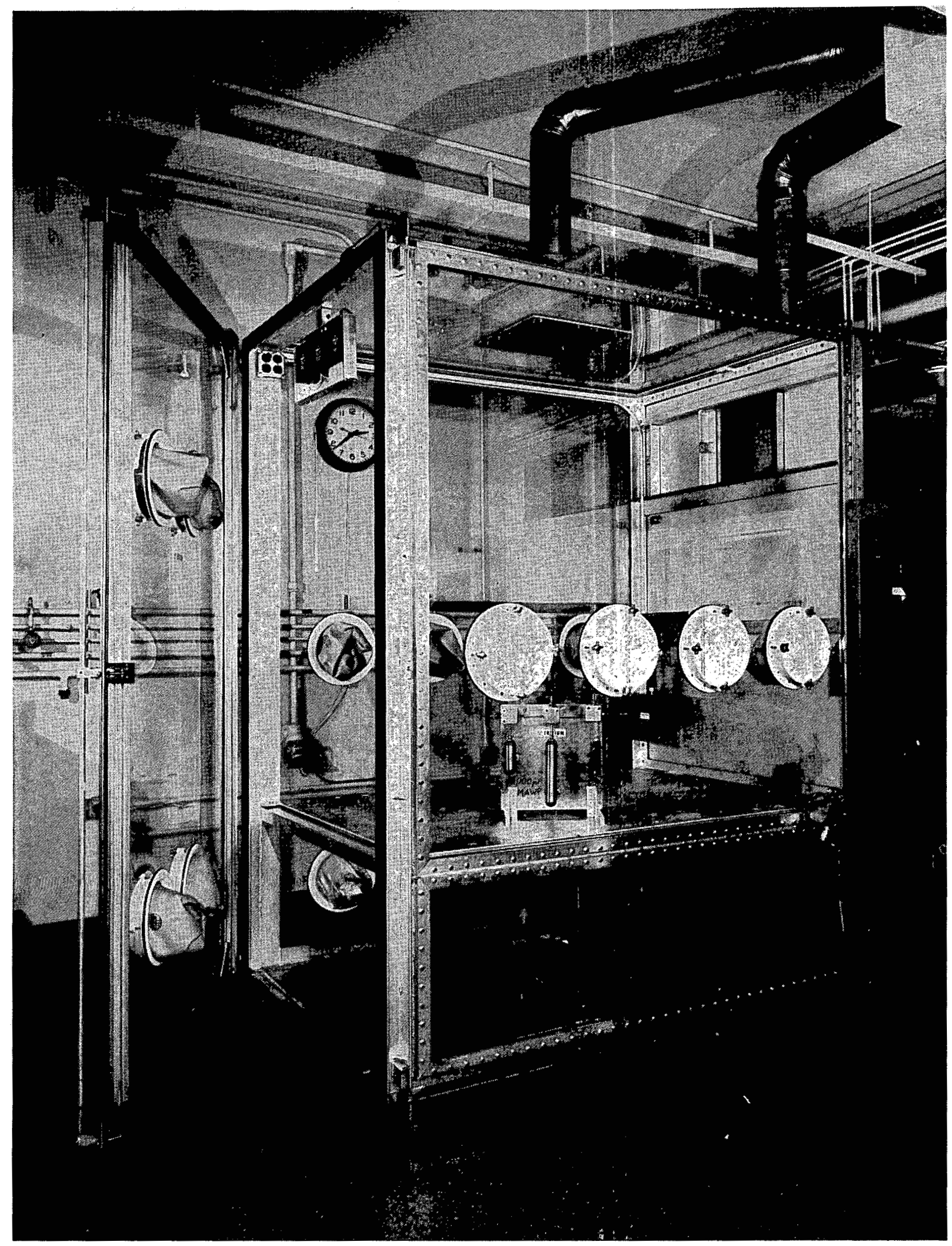

FIG. 2. Experimental enclosure. The large access door is shown partially open at the left. 




FIG. 3. Processing loop for experimental enclosure with instrumentation racks alongside. 


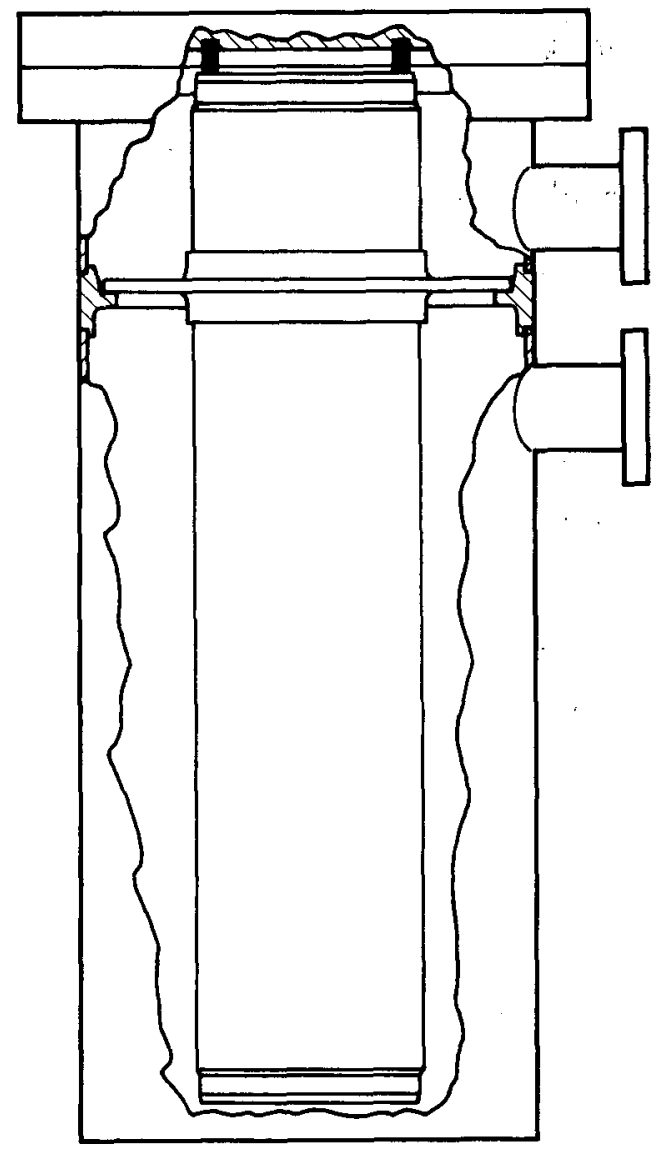

FIG. 4. Cutaway view of processing system canister. Removable packed bed cartridge rests on ledge, secured by small springs in top cover.

design to that used in the processing loop but with a little less capacity. Linde $5 \mathrm{~A}$ molecular seive adsorbent is used to dry the flowing air stream. The upstream ionization chamber measures total tritium concentration as gas and tritiated water, and the downstream ionization chamber (after the drying bed) measures the residual tritium gas after tritiated water or other adsorbable tritiated species are removed from the sampling line air flow.

\section{INSTRUMENTATION}

The ionization chambers used for the detection of tritium beta decay are Model 120 Betatec (2-l, autoranging) manufactured by Overhoff \& Associates.

Moisture measurements are made with Panametrics alumina capacitance-type probes. One probe is located downstream of the adsorbent bed in each loop to verify drying efficiency. Indicated moisture levels after drying are in the range of 1-10 parts per million by volume. Another probe at the processing 
loop inlet follows the change in enclosure moisture level during the course of an experimental run.

Pressure measurements are made at several locations in the experimental system using Teledyne Tabor Model 254 0-172 kPa absolute pressure transducers. The ionization chamber pressures differ by about 10-15, because of flow losses, which necessitates a correction with respect to the chamber concentration readings to put them on the same pressure base.

The primary temperature indicator for the enclosure atmosphere is a precision mercury-in-glass thermometer with $0.1 \mathrm{~K}$ graduations.

Flow rate in the processing loop is monitored with a Matheson Model 8164

mass flowmeter. As a check, this instrument was compared with a float-type meter and the two were found to agree within about 28.

TRITIUM GAS-PULSE INJECTOR

Controlled impulses of tritium gas are injected into the enclosure by means of a pressure vessel valved to a small volume trapped within a length of highpressure tubing. The injector, which can be seen in Fig. 2, rests within the enclosure and is operated through gloveports. One encl of the high-pressure tubing is connected to a valve exhausting into the encilosure. The other end is valved into a source of inert gas used to flush residual tritium from the tubing. Autoclave valves are used for the system. We introduced $50 \mathrm{Ci}$ of tritium gas into the pressure vessel initially, and diluted this by pressurization to 3.1 MPa with helium. The injector delivers about 0.1 Ci per pulse. 


\section{CATALYST CHARACTERIZATION}

We tested three different catalysts in this study. Each is in the form of cylindrical pellets composed of a low concentration of precious metal particles dispersed on a high-surface-area substrate. A description of each of the catalysts is given below, followed by a discussion of the quantitative characterization data. We identify the catalysts with a dual label: metal/substrate.

\section{Pd/KAOLIN CATALYST}

The Pd/kaolin catalyst is manufactured by Air products and Chemicals, Inc., identified as HSC-205, and described as a nominal 0.58 palladium on activated kaolin clay. This catalyst has not been tested before, as far as we know, in tritium service. The cylindrical pellets are a nominal 0.2 in. in diameter. The external surface is a light brown color, while the interior of the pellet is white with the brown coloration limited to a thin surface skin about 50-100 $\mathrm{mm}$ thick.

\section{Pt/ALUMINA CATALYST}

The Pt/alumina catalyst is manufactured by Englehard Minerals and Chemicals Corporation, identified as \#50088, and described as nominal 0.1-1.08 platinum on activated alumina. Performance test data are available on this catalyst. ${ }^{7}$ The cylindrical pellets are a nominal $1 / 8$ in. in diameter. The external pellet surface is a dark gray color with the coloration limited to a thin surface skin similar to the Pd/kaolin catalyst.

\section{Pd/ZEOLITE CATALYST}

The commercial catalysts discussed above are prepared by proprietary methods. We felt it would be useful to have a reference catalyst prepared by a method described in the literature. A Pd/zeolite catalyst was chosen, prepared by the method of Ostlund. ${ }^{8}$ This catalyst has been used by Ostlund in a mobile sampling system for the detection of atmospheric tritium. The preparation procedure may be summarized as follows: 
Dissolve $7.5 \mathrm{~g}$ of $\mathrm{dry}^{\mathrm{PdCl}_{2}}$ in $84 \mathrm{ml}$ of $\mathrm{IN} \mathrm{HCl}$ and add distilled water to obtain a clear reddish-brown solution. Cool to ambient temperature and adjust the volume to $245 \mathrm{ml}$ with distilled water. Pour the entire solution rapidly onto $600 \mathrm{~g}$ of $4 \mathrm{~A}$ zeolite molecular seive pellets and shake vigorously. Continue shaking until the charge has cooled and the pellets have a dry appearance and flow freely. The catalyst is now ready for drying and $\mathrm{H}_{2}$ activation. We followed this procedure, except for the substifution of Linde $5 \mathrm{~A}$ zeolite molecular seive pellets of nominal $1 / 16$ in. diameter. The pellets vary from a light brown to light gray color, and there is no distinct surface skin coloration as observed with the commercial catalysts.

\section{CATALYST PHYSICAL PROPERTIES}

Physical property measurements made on the three catalyst pellets are summarized in Table 1. Individual variations aside, all of the catalysts have large pore volumes and surface areas. Figure 5 shows the mercury penetration porosimeter data. There is probably significant additional pore volume present below the $15 \mathrm{~nm}$ pore-size limit of our porosimeter, especially for the Pd/kaolin catalyst whose pore volume curve is still rising steeply.

ELECTRON MICROPROBE SCANS

The distribution of precious metal on the catalyst pellet substrate is shown by the electron microprobe scans in Figs. 6, 7, and 8 . The scans start at the outer cylindrical surface of a sectioned pellet and proceed radially inward. The Pd/kaolin catalyst scan in Fig. 6 shows a rapid, exponential decrease in palladium concentration as the scan moves in from the outer surface of the pellet. The data soon reach the instrument noise level of about 0.18 by weight. A few additional data points taken closer to the center of the pellet confirm that there is no palladium impregnation beyond about $200 \mu \mathrm{m}$ from the outer surface.

The Pt/alumina catalyst scan in Fig. 7 shows a rapid, exponential decline in platinum concentration as the scan moves from the outer surface of the pellet toward the center. In contrast, a repeat scan with the instrument calibrated for palladium remains at instrument noise level at all radial 
TABLE 1. Catalyst physical properties.

\begin{tabular}{|c|c|c|c|c|}
\hline \multirow[b]{2}{*}{ Physical property } & \multirow[b]{2}{*}{ Units. } & \multicolumn{3}{|c|}{ Catalyst } \\
\hline & & Pd/kaolin & Pt/alumina & Pd/zeolite \\
\hline Pellet diameter ${ }^{a}$ & $\mathrm{~mm}$ & $4.44 \pm 0.05$ & $3.27 \pm 0.04$ & $1.64 \pm 0.08$ \\
\hline Pellet length ${ }^{a}$ & $\mathrm{~mm}$ & $4.71 \pm 0.75$ & $3.38 \pm 0.16$ & $2.89 \pm 1.04$ \\
\hline Equivalent sphere diameter ${ }^{b}$ & $\mathrm{~mm}$ & 4.53 & 3.31 & 1.92 \\
\hline Pellet density ${ }^{c}$ & $\mathrm{Mg} / \mathrm{m}^{3}$ & 1.280 & 1.601 & 1.124 \\
\hline Pellet pore volume ${ }^{C}$ & $\mathrm{~m}^{3} / \mathrm{Mg}$ & 0.245 & 0.122 & 0.300 \\
\hline Pellet surface area ${ }^{d}$ & $\mathrm{~m}^{2} / \mathrm{g}$ & 77 & 110 & 481 \\
\hline Bulk density & $\mathrm{Mg} / \mathrm{m}^{3}$ & 0.820 & 1.000 & 0.650 \\
\hline
\end{tabular}

${ }^{a}$ Mean value of a sample of 20 pellets. Variation shown is one standard deviation.

${ }^{b}$ Sphere with same external surface area/volume ratio as cylindrical pellet.

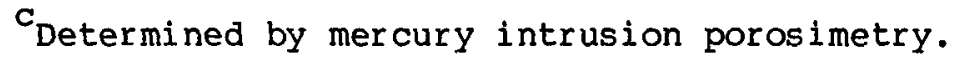

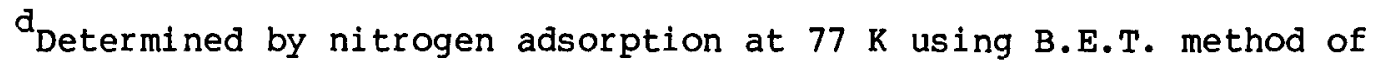
analysis.

positions. Bixel and Kershner report ${ }^{7}$ that this catalyst contains both platinum and palladium. We find no detectable palladium above background.

The two commercial catalysts are similar in that their precious metal content is confined to a thin surface layer. According to Wei ${ }^{9}$ this is a conventional policy of catalyst design, the so-called eggshell catalyst. In contrast, Ostlund's method of impregnation of the Pd/zeolite catalyst leads to a more uniform deposition of the precious metal as shown in Fig. 8 . We have drawn a line through the data, although the exponential approximation is not nearly as good as in the other two scans.

Table 2 summarizes the data derived from the electron microprobe scans. The concentration e-folding depth for the commercial catalysts is consistent with the 50-100 $\mu \mathrm{m}$ visually observed depth of skin coloration. Integration over the volume of the catalyst pellet leads to an average of $0.12,0.20$, and 0.83 percent by weight of precious metal for $\mathrm{Pd} /$ kaolin, $\mathrm{Pt} / \mathrm{alumina}$, and $\mathrm{Pd} /$ 


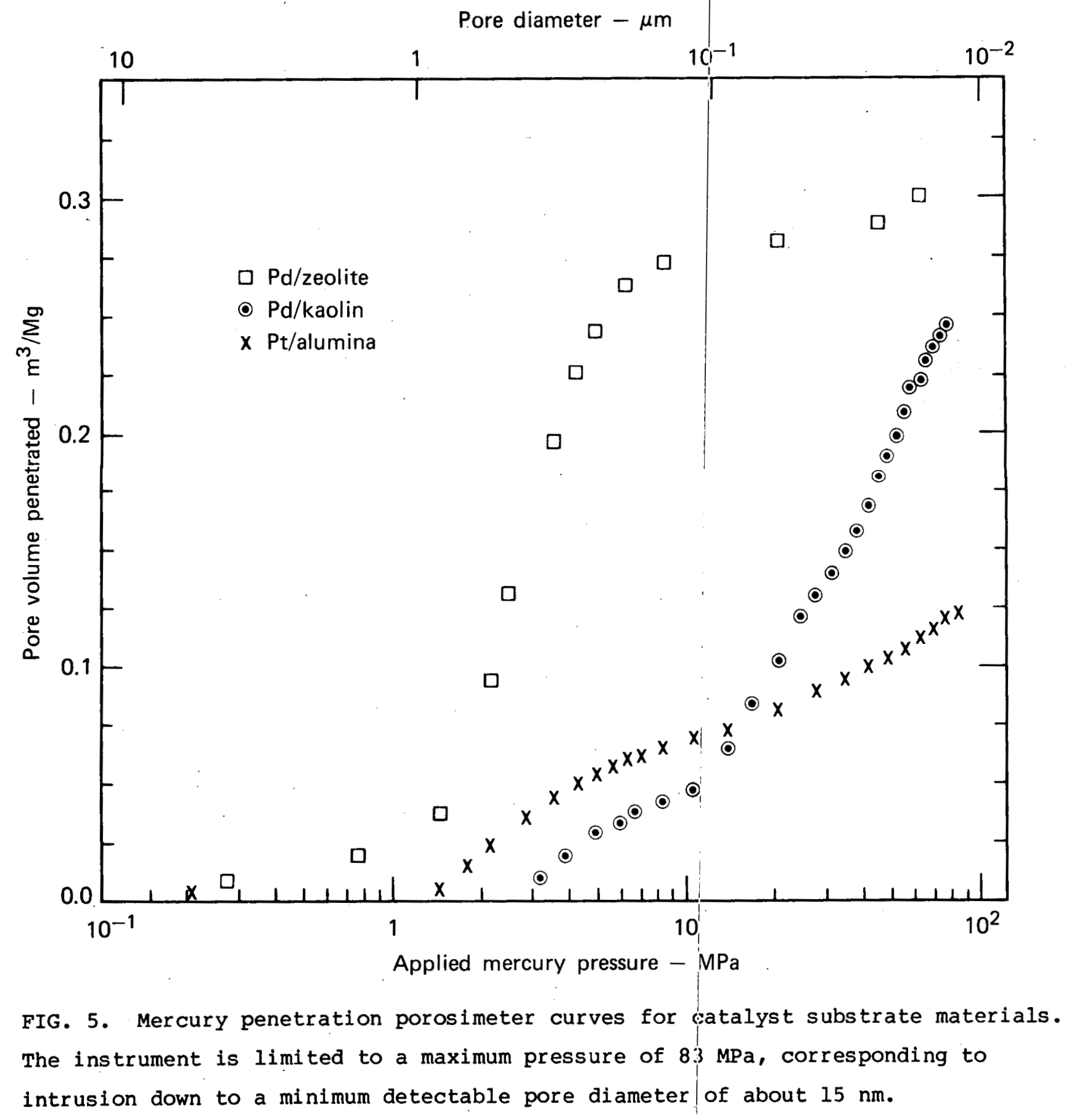




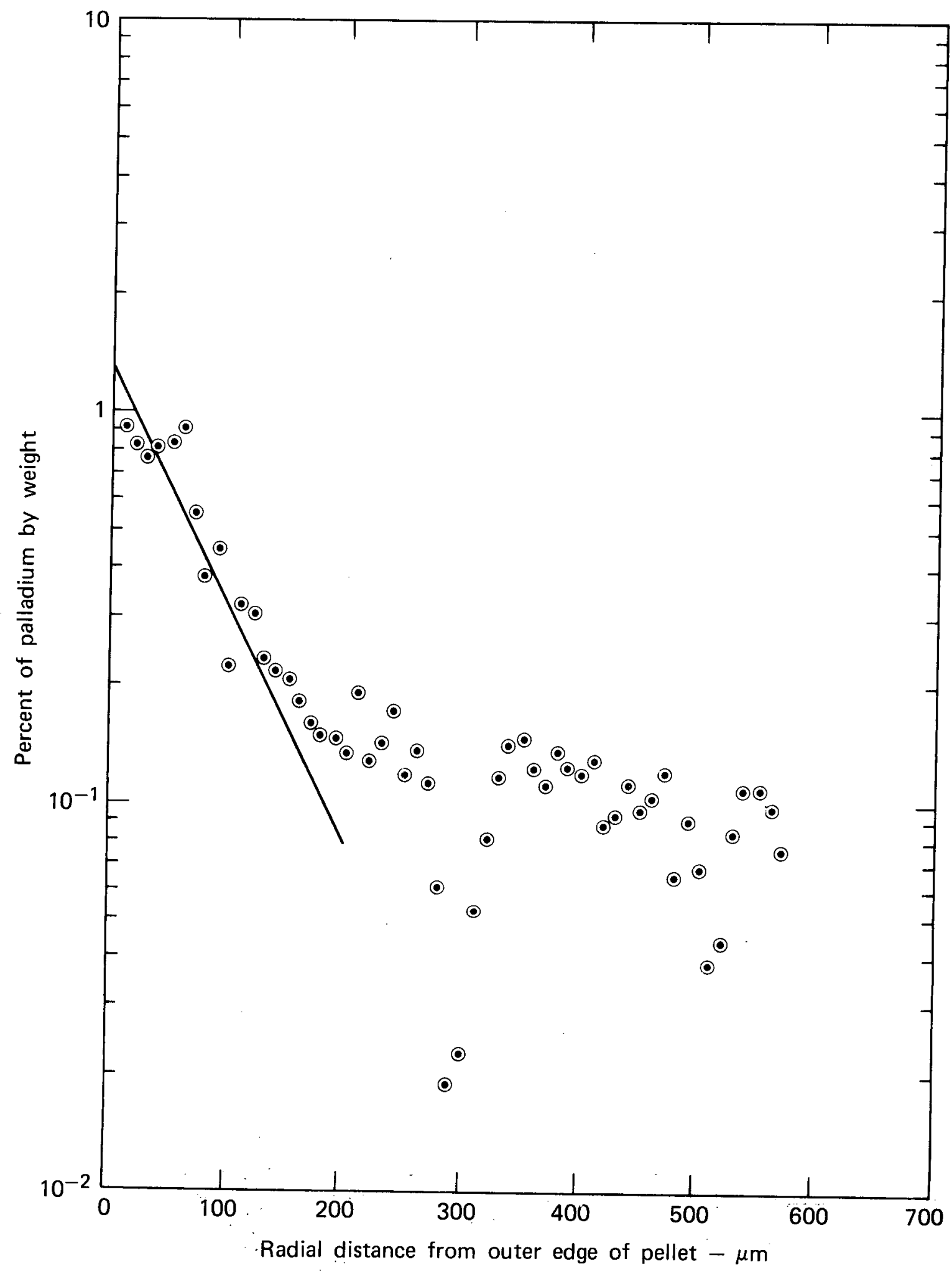

FIG. 6. Electron microprobe scan of $\mathrm{Pd} /$ kaolin pellet. 


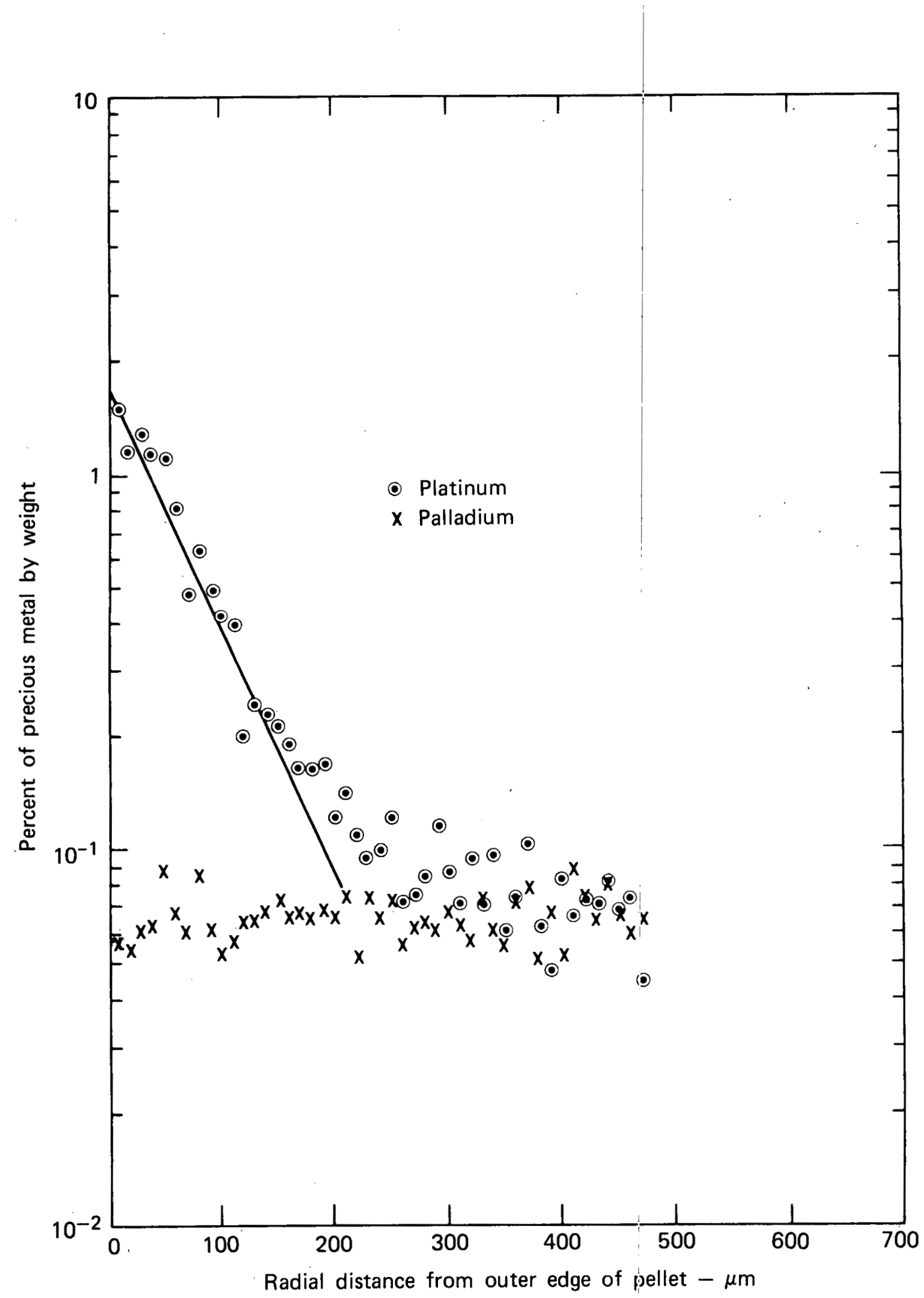

FIG. 7. Electron microprobe scans of Pt/alumina peliet. Response to palladium is at the instrument noise level. 


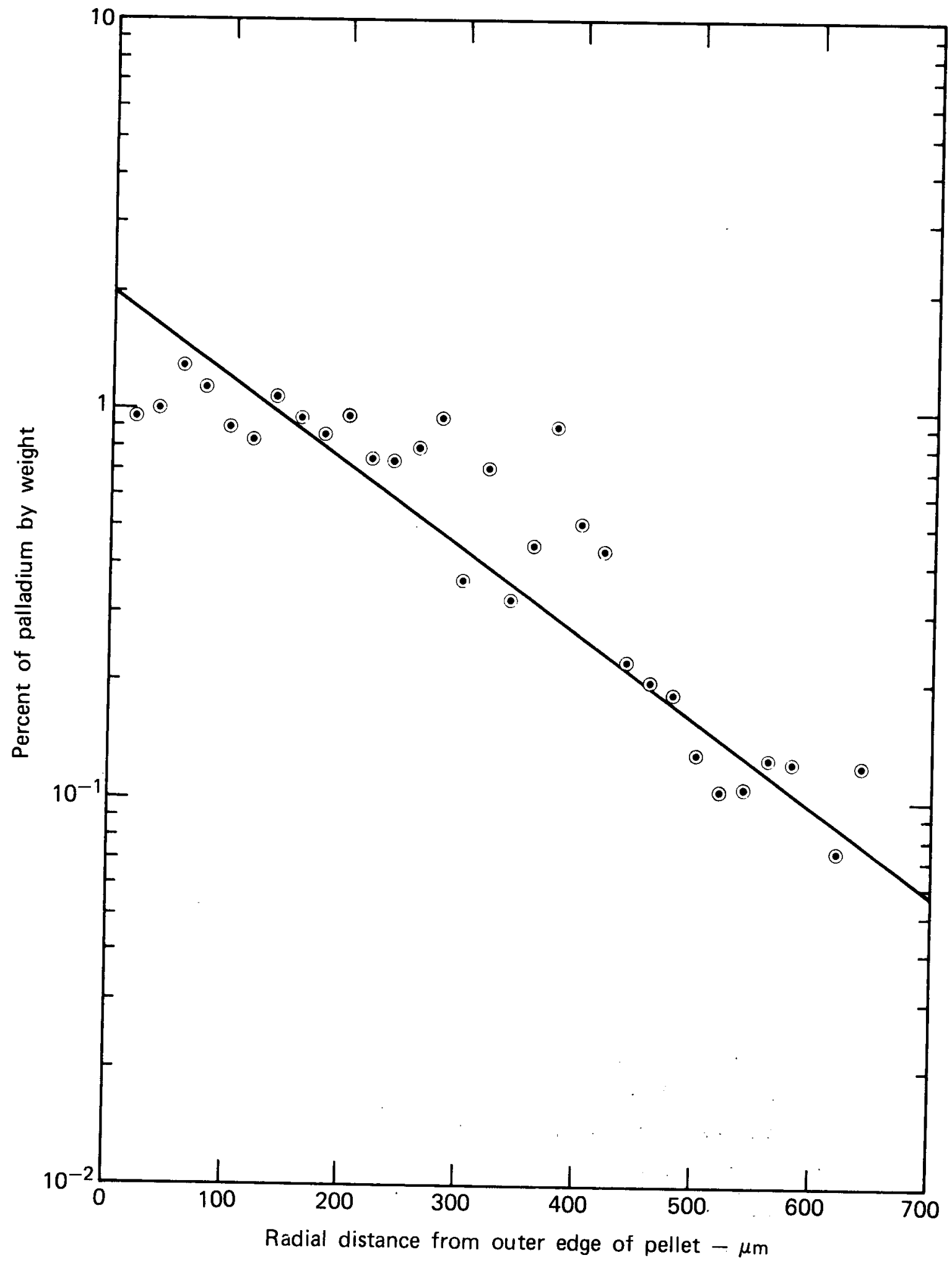

FIG. 8. Electron microprobe scan of $\mathrm{Pd} /$ zeolite pellet. 
TABLE 2. Characteristics of the dispersed precious metal on the catalyst substrate.

\begin{tabular}{|c|c|c|c|c|c|}
\hline \multirow[b]{2}{*}{ Property } & \multirow[b]{2}{*}{ Units } & \multicolumn{4}{|c|}{ Caitalyst } \\
\hline & & $\mathrm{Pd} / \mathrm{kaolin}$ & Pt/ & alumina & $\mathrm{Pd} /$ zeolite \\
\hline $\begin{array}{l}\text { Pellet-surface metal } \\
\text { concentration }\end{array}$ & wto & 1.3 & & 1.8 & 2.0 \\
\hline $\begin{array}{l}\text { Concentration e-folding } \\
\text { depth }\end{array}$ & $\mu \mathrm{m}$ & 72 & & 66 & 198 \\
\hline $\begin{array}{l}\text { Calculated volume-average } \\
\text { metal concentration }\end{array}$ & wto & 0.12 & & 0.20 & 0.83 \\
\hline $\begin{array}{l}\text { Measured pellet average } \\
\text { metal concentration }\end{array}$ & wtz & - & & - & 0.75 \\
\hline $\begin{array}{l}\text { Calculated dispersed metal } \\
\text { surface area per pellet mass }\end{array}$ & $m^{2} / g$ & 0.59 & & 0.56 & 3.7 \\
\hline
\end{tabular}

abtained from the intercept of the lines drawn on the electron microprobe radial scans.

bepth at which the concentration has decreased from the surface value by a factor $1 / e$.

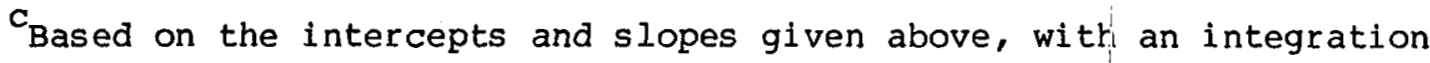
over the equivalent spheres with diameters given in Taible 1.

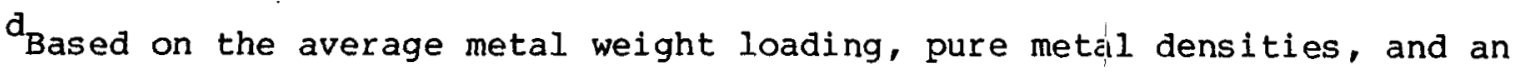
average dispersed metal particle size of $1 \mathrm{~nm} .^{10}$

zeolite, respectively. For the Pd/zeolite pellets we know that the correct average composition is 0.758 by welght based on the proportions used in preparation, and so the calculated composition is about $10 \%$ high. This minor error for the calculated volume-average composition lends confidence to the calculated average metal compositions for the commercial catalysts.

Finally, in Table 2 we make a rough estimate of the available dispersed metal surface area based on an assumed particle size 10 of $1 \mathrm{~nm}$. From the cited reference, it appears that this is a likely average particle size within a factor of two. The surface area estimate will also be in error from the estimated weight loading. We conjecture that the estimated metal surface area 
may be correct within a factor of 3. The reason for recording this rough estimate is that it will be useful in a later section of this report when making comparisons with kinetic data that are based on surface area.

\section{CATALYST ACTIVATION}

We followed a consistent procedure to activate the catalysts prior to their use in the experimental tests. A charge of catalyst pellets was sealed in a small steel reactor with flow-through connections, and a stream of hydrogen gas was passed through the pellets. The reactor was brought up to a temperature of about $500 \pm 100 \mathrm{C}$ and held there for about $6 \mathrm{~h}$, then allowed to cool overnight in a continuing flow of hydrogen gas. The activated catalyst was then loaded into place in the processing loop. 
PROCEDURE AND METHOD OF ANALYSIS

The general procedure for an experimental run is as follows:

Secure enclosure door, start enclosure air-circulation fans, start flow through processing and sampling loops and $r$ un for a few minutes until instrument readings stabilize at base levels. Inject tritium pulse and record subsequent concentration decay along with other instrumental data. At the end of the $r$ un, vent enclosure atmosphere to exhaust system, and flush air through sampling loop to obtain postexperiment background reading on ion chambers.

To facilitate discussion of the experimental $r$ uns, we next outline the method of data analysis. The first step is to determine fractional conversion of the catalytic converter. Next, we examine the structure within the converter and determine the kinetic rate constant for the catalyst.

\section{CATALYST BED FRACTIONAL CONVERSION}

We make the following assumptions about the enclosure and processing system:

1. Well-mixed enclosure air space, volume $v$, intially at tritium concentration $\mathrm{C}_{0}$.

2. Processing system recirculates air at constant flow rate Q.

3. Catalyst bed converts a fixed fraction, $f$, of: input tritium gas to water vapor (this implies a first-order reaction).

4. Adsorbent bed captures all tritiated water formed, and passes all unreacted tritium gas.

5. Negligible permeation of tritium to/from walls of enclosure.

6. Negligible uncatalyzed gas/water conversion within the enclosure.

Assumption 1 holds after about a ten-minute lag Eor mixing following tritium injection. The constant flow rate of assumption 2 is easily maintained within a few percent. Assumption 3, a fixed ffactional conversion per pass through the reactor, implies a first-order chemical reaction. The data to be presented confirm this assumption.

Conversion in the catalyst bed does not depend on subsequent tritiated water adsorption. For purposes of analysis, however, we need a measurement of 
the change of tritium gas concentration with time. We therefore require the efficient water adsorbent bed of assumption 4 since an ionization chamber cannot, per se, distinguish between tritium gas and tritiated water vapor. We will return to this assumption when discussing the experimental data.

Assumptions 5 and 6 are confirmed by the data, at least within the time scale and concentration range of interest in this report.

Using these assumptions, we find the mass balance for tritium within the enclosure leads to an exponential decline of concentration with time:

$$
C=C_{0} \exp (-f Q t / V)
$$

The catalyst-bed fractional conversion, $f$, can be determined from the logarithmic slope of the enclosure concentration decay curve:

$$
f=\frac{v}{Q}\left(-\frac{d \ln c}{d t}\right) \text {. }
$$

KINETIC RATE CONSTANT DETERM INATION

To translate fractional conversion data into meaningful information on catalyst kinetics, we introduce a catalyst-bed model based on the following assumptions:

- Pseudohomogeneous first-order reaction with rate constant $k$.

- Constant flow rate, $Q$, through a catalyst bed of volume $V_{c}$.

- Isothermal reactor.

- Negligible radial concentration gradients (implies particle diameter $<$ reactor diameter).

- Negligible axial dispersion (implies reactor length > particle diameter).

- Steady-state operation within reactor.

These assumptions are all reasonable. Further discussion with references to literature sources is available in an earlier review paper. ${ }^{6}$

Using the foregoing assumptions, the mass balance for tritium within the catalytic reactor leads to the following expression for fractional conversion:

$$
\mathrm{f}=1-\exp \left(-\mathrm{KV}_{\mathrm{c}} / Q\right)
$$


Since $f$ has been determined experimentally, we can caleulate the kinetic rate coefficient:

$$
R=\frac{Q}{V_{c}} \ln \left(\frac{1}{1-f}\right)
$$

The rate coefficient, $K$, is an intrinsic property of a particular catalyst at the operating temperature, at least in first approximation and of ten to a very good approximation. We will estimate the more fundamental surface rate constant in a subsequent section. 
We will first show typical data on enclosure drying characteristics and then present data from each catalyst test.

ENCLOSURE DRYING CHARACTERISTICS

Moisture levels in the enclosure during an experimental run generally follow the behavior shown in Fig. 9, with a few exceptions that will be noted later. For the first hour or so, the enclosure moisture level drops exponentially with a logarithmic slope corresponding to essentially complete removal per pass through the adsorbent bed. Subsequent drying becomes sluggish because of moisture outgassing from the enclosure walls. 11

We conclude, from the data in Fig. 9 and the test data that follow, that catalytic rate behavior is insensitive to moisture level, at least in the 100-4000 ppm (parts per million) range. Catalyst test data cover different time scales, and variations in moisture level apparently have no noticeable effect.

The adsorbent-bed outlet air drops immediately to a low moisture level and remains essentially constant throughout the run. The adsorbent bed between the two ion chambers in Fig. 1 also shows a very low outlet molsture level, which gives us confidence that the downstream ion chamber sees very little, if any, tritiated water vapor.

\section{Pd/KAOLIN (UNACTIVATED)}

The catalyst activation procedure that we described earlier (p. 17) was inspired by our first experimental run, shown in Fig. 10, which was carried out with the Pd/kaolin catalyst in as-recelved condition. There was no measurable catalytic oxidation of tritium over a 3-hour period.

In addition to demonstrating the necessity for activation, this run is important for several reasons. First, it shows that mixing time is about 10 to $20 \mathrm{~min}$ after tritium is injected into the enclosure.

Figure 10 also shows a discrepancy of about 68 in the ion chamber readings, with the "dry" chamber reading higher than the "wet" chamber. We believe this difference is caused by imprecise electronic amplification, 


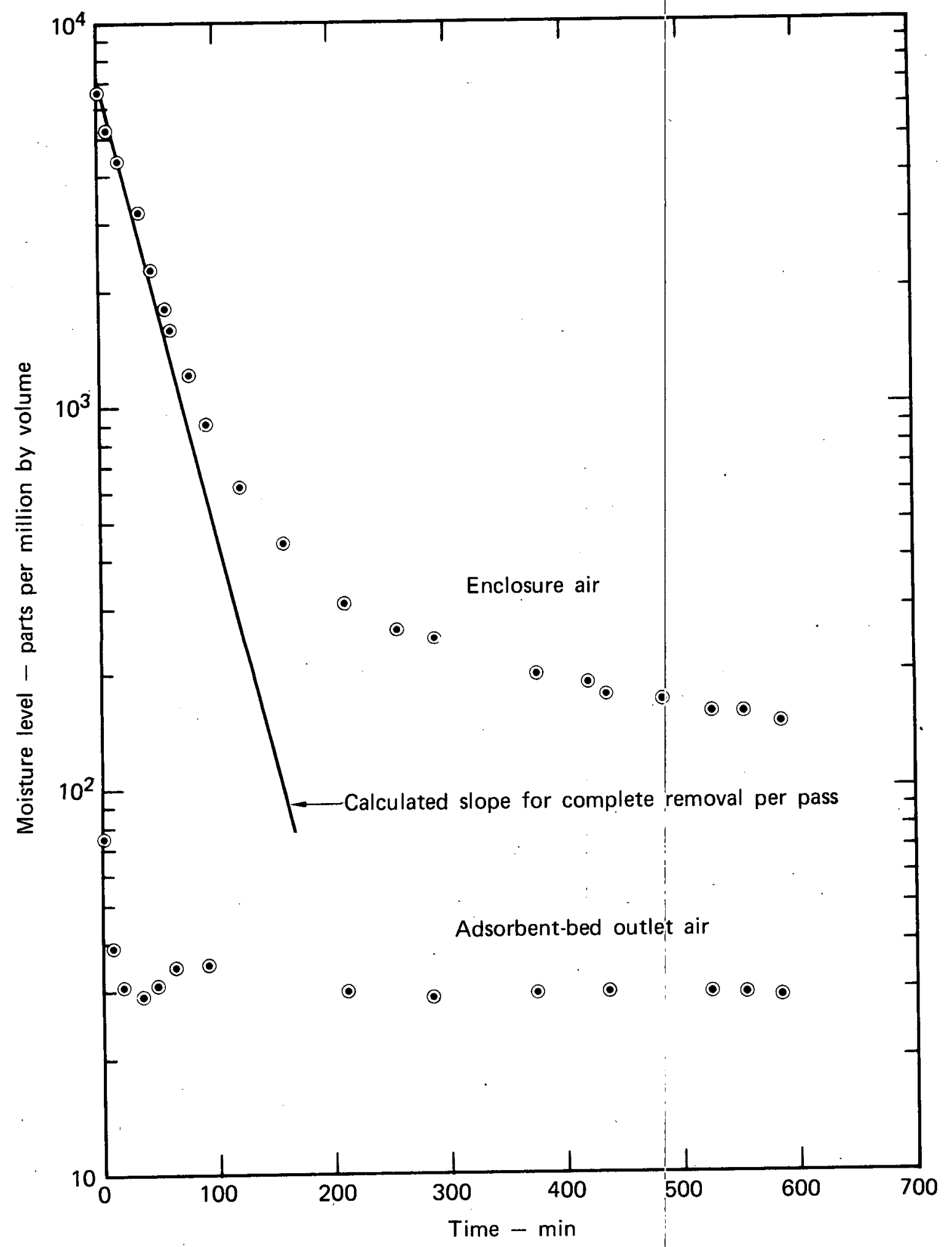

FIG. 9. Typical behavior of moisture levels during experimental run. Enclosure moisture follows calculated curve for about an hour and then begins to tail off because of moisture outgassing from the Lucite walls. Adsorbent-bed outlet moisture drops quickly to a low level and remains nearly constant thereafter. 


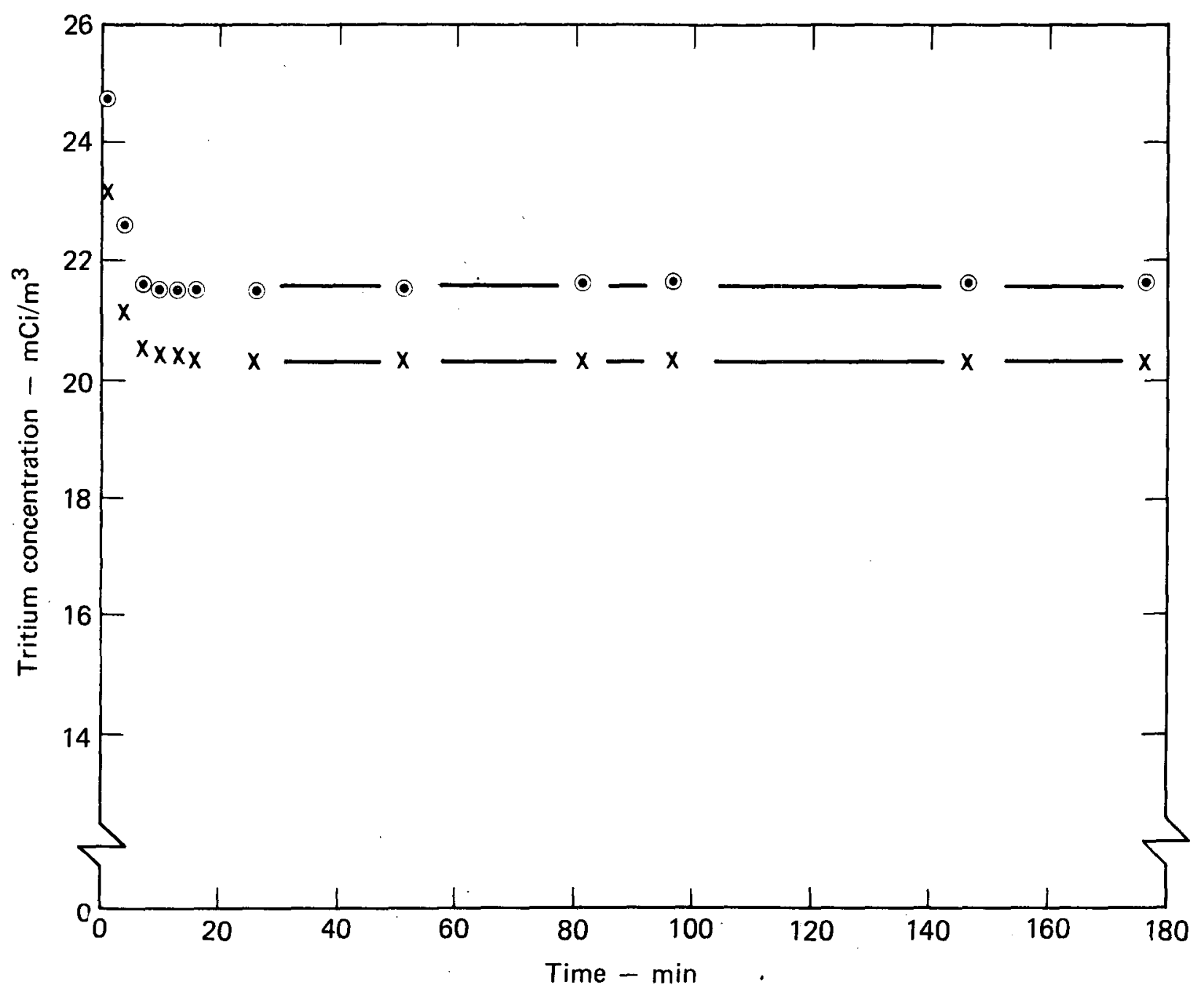

FIG. 10. Tritium/air catalytic oxidation with unactivated catalyst. No measurable reaction with unactivated Pd/kaolin. The 68 difference between upper curve (dry chamber) and lower curve (wet chamber) is instrument inaccuracy. 
since chamber pressures were measured and corrections we also observed abrupt shifts of about 58 in a given chamber when shifting from one scale range to another.

Perhaps the most important information gained from Fig. 10 is that no appreciable disappearance of tritium gas by permeation loss or uncatalyzed gas/water conversion occurs over several hours. We can therefore attribute the observed conversion in later runs to catalytic activity.

Pd/KAOLIN (AGE $=0.1 \mathrm{~d})$

A fresh batch of Pd/kaolin catalyst was activated in hot hydrogen. After overnight cooling, the catalyst was removed from the hydrogen atmosphere and testing began within a few hours.

The test results are shown in Fig. 11, and there is a dramatic improvement over the unactivated state of Fig. 10. The most impoltant feature of Fig. 11 is the linear slope during the midportion of the run, demonstrating a first-order reaction.

Wet and dry ton chamber readings are nearly identical until late in the run when the wet-chamber reading starts to deviate from the linear slope. The difference between the readings may indicate that a small amount of tritiated water is present within the enclosure, either formed there by isotopic exchange or fed back from the processing loop by escape from the absorbent bed. The dry ion chamber reading eventually levels out near instrument background.

The straight-line conversion slope drawn through the data has a numerical value of $0.0173 \mathrm{~min}^{-1}$. Table 3 shows the data for the run, including a calculated conversion slope for each consecutive pair of data points. It can be seen that these calculated slopes agree well with the straight-line average slope drawn on the graph. We also.show in Fig. 11 the calculated concentration decay curves that would prevail for zero- and second-order reactions,

\footnotetext{
*We assign an "age" to each catalyst test. By this/we mean the number of days of exposure to air between activation of the batch and the catalytic conversion run. All three catalysts, as it turns out, degrade with air exposure, and so age is an important parameter.
} 


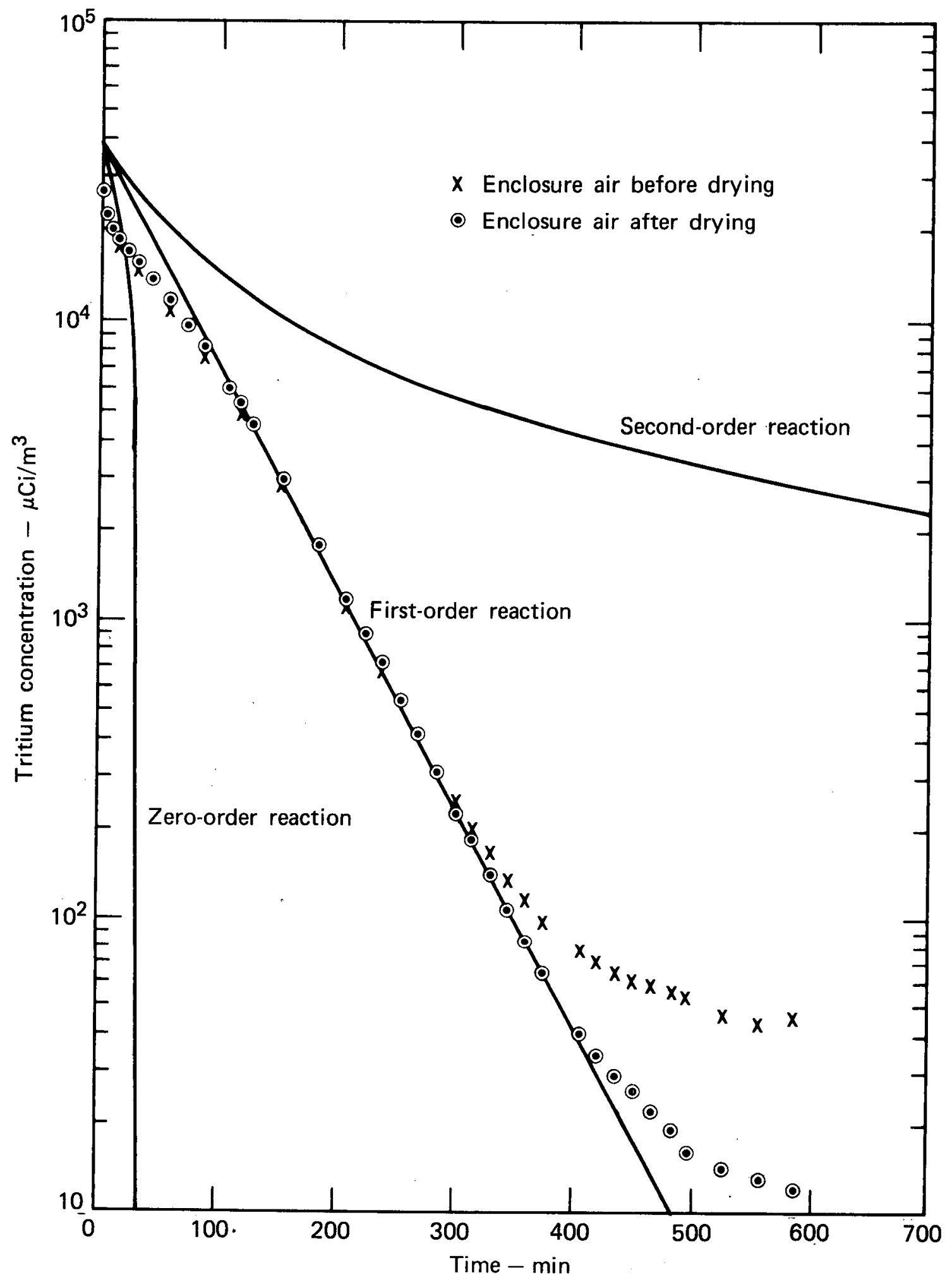

FIG. 11: Tritium/air catalytic oxidation with Pd/kaolin (age $=0.1$ d). Conversion slope $=0.0173 \mathrm{~min}^{-1}$. Fractional conversion $=0.646$. Rate coefficient $=1.75 \mathrm{~s}^{-1}$. 
TABLE 3. Tritium removal from enclosure atmosphere (Pd/kaolin oxidation catalyst, zeolite adsorbent). ${ }^{a}$

\begin{tabular}{|c|c|c|c|c|}
\hline \multirow[b]{2}{*}{$\begin{array}{l}\text { Time } \\
\text { (min) }\end{array}$} & \multicolumn{2}{|c|}{$\begin{array}{l}\text { Concentration } \\
\left(\mu \mathrm{Ci} / \mathrm{m}^{3}\right)\end{array}$} & \multicolumn{2}{|r|}{$\begin{array}{c}-d \ln c / d t \\
\left(\min ^{-1}\right)\end{array}$} \\
\hline & $\begin{array}{c}\text { Enclosure } \\
\text { air }^{b}\end{array}$ & $\begin{array}{l}\text { Desiccated } \\
\text { air }^{c}\end{array}$ & $\begin{array}{r}\text { Enclosu } \\
\text { air }\end{array}$ & $\begin{array}{c}\text { Desiccated } \\
\text { air }\end{array}$ \\
\hline-2 & 1 & 10 & - & - \\
\hline 0 & (inject tritil & um spike) & - & - \\
\hline 4 & $2.46 \times 10^{4}$ & $2.75 \times 10^{4}$ & - & - \\
\hline 7 & $2.10 \times 10^{4}$ & $2.26 \times 10^{4}$ & 0.053 & 0.065 \\
\hline 11 & $1.90 \times 10^{4}$ & $2.00 \times 10^{4}$ & 0.025 & 0.031 \\
\hline 17 & $1: 76 \times 10^{4}$ & $1.87 \times 10^{4}$ & $0.013^{1}$ & 0.011 \\
\hline 25 & $1.60 \times 10^{4}$ & $1.70 \times 10^{4}$ & 0.012 & 0.012 \\
\hline 33 & $1.47 \times 10^{4}$ & $1.57 \times 10^{4}$ & 0.011 & 0.010 \\
\hline 45 & $1.29 \times 10^{4}$ & $1.37 \times 10^{4}$ & 0.011 & 0.011 \\
\hline 60 & $1.09 \times 10^{4}$ & $1.16 \times 10^{4}$ & 0.011 & 0.011 \\
\hline 75 & $9.10 \times 10^{3}$ & $9.72 \times 10^{3}$ & 0.012 & 0.012 \\
\hline 90 & $7.50 \times 10^{3}$ & $8.05 \times 10^{3}$ & $0.013_{1}^{\prime}$ & 0.013 \\
\hline 110 & $5.54 \times 10^{3}$ & $5.97 \times 10^{3}$ & 0.015 & 0.015 \\
\hline 120 & $4.85 \times 10^{3}$ & $5.23 \times 10^{3}$ & 0.013 & 0.013 \\
\hline 130 & $4.12 \times 10^{3}$ & $4.46 \times 10^{3}$ & 0.016 & 0.016 \\
\hline 156 & $2.74 \times 10^{3}$ & $2.96 \times 10^{3}$ & 0.016 & 0.016 \\
\hline 185 & $1.68 \times 10^{3}$ & $1.79 \times 10^{3}$ & $0.01 \%$ & 0.017 \\
\hline 210 & $1.09 \times 10^{3}$ & $1.15 \times 10^{3}$ & 0.017 & 0.018 \\
\hline 225 & 836 & 881 & $0.01 \&$ & 0.018 \\
\hline 240 & 671 & 710 & 0.015 & 0.014 \\
\hline 255 & 523 & 538 & 0.017 & 0.018 \\
\hline 270 & 408 & 411 & 0.017 & 0.018 \\
\hline 285 & 318 & 310 & 0.017 & 0.019 \\
\hline 302 & 241 & 225 & 0.016 & 0.019 \\
\hline 315 & 201 & 182 & 0.014 & 0.016 \\
\hline 330 & 163 & 140 & 0.014 & 0.017 \\
\hline 345 & 132 & 105 & 0.014 & 0.019 \\
\hline 360 & 114 & -83 & 0.010 & 0.016 \\
\hline 375 & 96 & 64 & $0.01]$. & 0.017 \\
\hline 406 & 76 & 40 & 0.008 & 0.015 \\
\hline 420 & 70 & 34 & 0.006 & 0.012 \\
\hline 435 & 64 & 29 & 0.006 & 0.011 \\
\hline 450 & 60 & 26 & 0.004 & 0.007 \\
\hline 465 & 58 & 22 & 0.002 & 0.011 \\
\hline 483 & 55 & 19 & 0.003 & 0.008 \\
\hline 495 & 53 & 16 & 0.003 & 0.014 \\
\hline 525 & 46 & 14 & 0.005 & 0.004 \\
\hline 555 & 43 & 13 & 0.002 & 0.002 \\
\hline 585 & 45 & 12 & -0.002 & 0.003 \\
\hline 602 & (flush enclosure & atmosphere) & - & - \\
\hline 650 & 0 & 0 & - & - \\
\hline
\end{tabular}

axperimental setup shown in Fig. 1. Flow rate during this $r$ un was $146 \mathrm{l} / \mathrm{min}$ at $25 \mathrm{C}, 100.6 \mathrm{kPa}$. Enclosure temperature sitarted at. $23 \mathrm{C}$, increased to $25 \mathrm{C}$ at about $150 \mathrm{~min}$ and then remained constant.

bIon chamber corrected for zero offset by adding $-4 \mathrm{~h} \mathrm{Ci} / \mathrm{m}^{3}$ to reading.

CIon chamber corrected for zero offset by adding $+54 \mu \mathrm{\mu i} / \mathrm{m}^{3}$ to reading. Pressure correction multiplier of 1.110 app]ied to put ion chamber readings on the same pressure basis. 
starting from the same initial rate as the first-order reaction. It is evident that a first-order reaction is the most plausible choice.

Table 4 shows a summary of the data from this and subsequent runs. Fractional conversion is 64.68 per pass through the catalyst bed. The rate constant, estimated from the conversion and other data, is $1.75 \mathrm{~s}^{-1}$.

Pt/ALUMINA (AGE $=3$ d)

Test results for the $\mathrm{Pt} /$ alumina catalyst, activated and then aged three days in air, are shown in Fig. 12. Concentration readings drop abruptly at 35 minutes into the run from an instrument scale change. It can be seen that two displaced lines with the same slope fit the data quite well. A change of scale also occurs on Fig. Il at about the same concentration level but, for reasons unknown, does not appear particularly noticeable there. The conversion slope is particularly critical in this run. The slope, as drawn, corresponds to 99.28 conversion per pass. One could increase the slope of the line by about 58 without doing any injustice to the data, and thereby calculate more than $100 \%$ conversion per pass. If we assume a $\pm 5 \%$ error in the estimated slope, then the conversion may be as low as 948 . All that can be said about the upper limit is that it may be very close to $100 \%$.

The rate constant for this run is given in Table 4 as $7.73 \mathrm{~s}^{-1}$. It is apparent from Eq. (4) that the relative error in the rate constant will increase as the fractional conversion approaches unity. This difficulty may be circumvented by removing part of the catalyst charge from the reactor in order to reduce the conversion, or by increasing the flow rate.

Pt/ALUMINA (AGE $=17 \mathrm{~d})$

We discovered that another method of reducing conversion in the catalytic oxidizer is to allow the catalyst to age in air for a while and then to repeat the test. Results are shown in Fig. 13 for the Pt/alumina catalyst at 17 days of air exposure. The calculated results are summarized in Table 4, which shows that conversion has dropped to 80.68 and the corresponding rate constant is $2.64 \mathrm{~s}^{-1}$.

Through Figs. 11, 12, and 13 one can see a progressive upward trend in the residual concentrations at the end of each run. This is caused primarily by the generation of tritiated methane in our steel-walled tritium injector, 
TABLE 4. Summary of experimental data ${ }^{a}$ and calculated results. ${ }^{b}$

\begin{tabular}{|c|c|c|c|c|c|c|c|}
\hline $\begin{array}{l}\text { Catalyst/ } \\
\text { substrate }\end{array}$ & $\begin{array}{l}\text { Age } \\
\text { (d) }\end{array}$ & $\begin{array}{l}\text { Conversion } \\
\text { slope } \\
\left(\min ^{-1}\right)\end{array}$ & $\begin{array}{c}\text { Flow rate } \\
(\ell / \mathrm{min})\end{array}$ & $\begin{array}{l}\text { Temperature } \\
\text { (C) }\end{array}$ & $\begin{array}{l}\text { Fractional } \\
\text { conversion }\end{array}$ & $\begin{array}{l}\text { Catalyst } \\
\text { volume } \\
\text { (l) }\end{array}$ & $\begin{array}{c}\text { Rate } \\
\text { coefficient } \\
\left(\mathrm{s}^{-1}\right)\end{array}$ \\
\hline $\mathrm{Pd} /$ kaolin & 0.1 & 0.0173 & 146 & 25 & 0.646 & 1.44 & 1.75 \\
\hline $\mathrm{Pd} /$ kaolin & 336 & 0.00306 & 152 & 27 & 0.110 & 1.44 & 0.205 \\
\hline $\mathrm{Pd} /$ kaolin ${ }^{\mathrm{C}}$ & 2 & 0.0165 & 157 & 24 & 0.573 & 1.40 & 1.59 \\
\hline Pt/alumina & 3 & 0.0264 & 145 & 24 & 0.992 & 1.51 & 7.73 \\
\hline Pt/alumina & 17 & 0.0216 & 146 & 25 & 0.806 & 1.51 & 2.64 \\
\hline Pt/alumina & 380 & 0.00890 & 151 & 25 & 0.321 & 1.51 & 0.645 \\
\hline $\mathrm{Pd} /$ zeolite & 1 & 0.0265 & 151 & 24 & 0.956 & 0.526 & 14.9 \\
\hline $\mathrm{Pd} /$ zeolite & 35 & 0.0121 & 154 & 24 & 0.428 & 0.526 & 2.73 \\
\hline
\end{tabular}

${ }^{a}$ Conversion slope $=(-d l n c / d t)$.

Fractional conversion calculated from Eq. (2) with an enclosure volume of 5450 t. Catalyst volume based on weight and the bulk density given in Table l. Kinetic rate coefficient calculated from Eq. (4).

$\mathrm{C}_{\text {Tested }}$ after reactivation. 


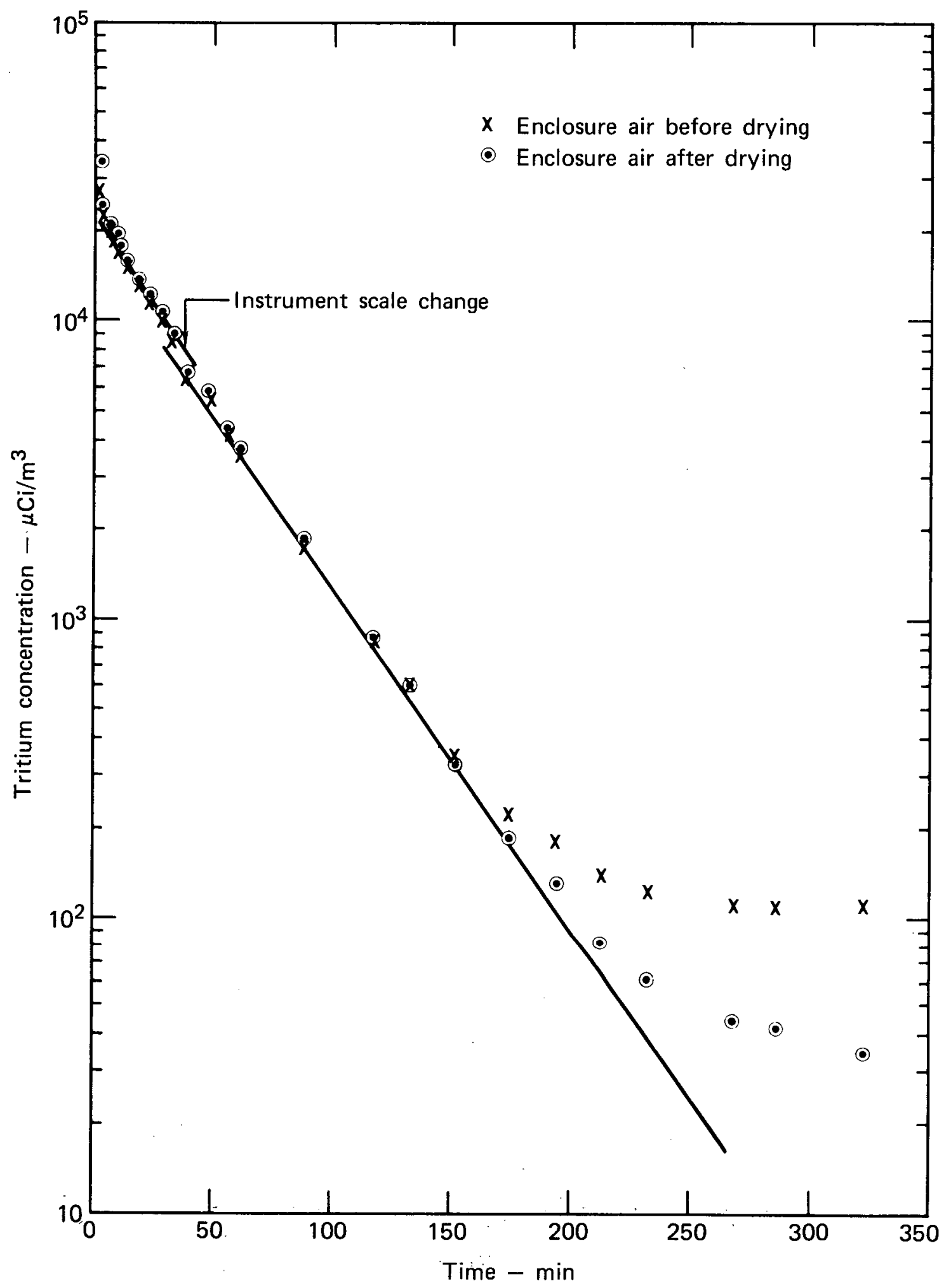

FIG. 12. Tritium/air catalytic oxidation with Pt/alumina (age = 3 d). Conversion slope $=0.0264 \mathrm{~min}^{-1}$. Fractional conversion $=0.992$. Rate coefficient $=7.73 \mathrm{~s}^{-1}$. 


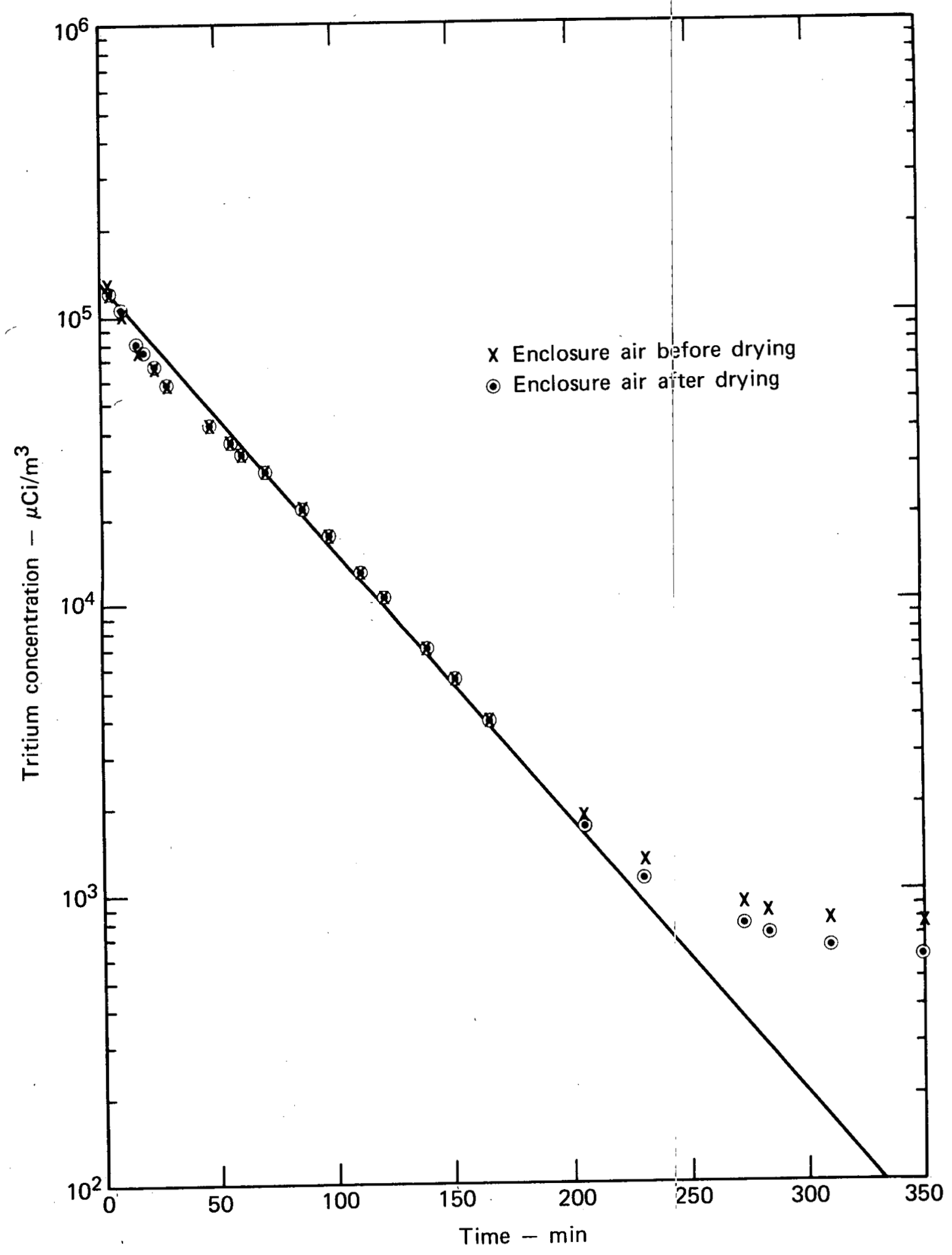

FIG. 13. Tritium/air catalytic oxidation with Pt/alumina (age $=17 \mathrm{~d}$ ). Conversion slope $=0.0216 \mathrm{~min}^{-1}$. Fractional conversion $=0.806$. Rate coefficient $=2.64 \mathrm{~s}^{-1}$. 
as shown by analytical data presented later in this report. There may be some increase in residual tritiated water in Fig. 13 compared to Fig. 12, and this would be consistent with the higher initial tritium gas concentration shown in Fig. 13. The apparent increase may be spurious, however, since it is based on the difference between wet- and dry-chamber readings, which is relatively small and therefore inaccurate.

Pd/KAOLIN $(A G E=336 \mathrm{~d})$

The $\mathrm{Pd} /$ kaolin catalyst was rerun after nearly one year of exposure to air. Catalyst performance deteriorated considerably compared to the test results discussed above for freshly activated catalyst. We attempted to reactivate the catalyst in situ by injecting $5 \mathrm{l}$ of hydrogen gas into the enclosure. We were unable to introduce a larger quantity because of pressure limitations of the enclosure. Nevertheless, the amount introduced corresponds to about $920 \mathrm{ppm}$ (parts per million by volume) and is large compared to ambient hydrogen concentration in air $(0.5 \mathrm{ppm})$. The amount of hydrogen injected results in a concentration very much higher than the initial tritium concentration in a typical experiment since $10^{5} \mu \mathrm{Ci} / \mathrm{m}^{3}$ is equivalent to $0.04 \mathrm{ppm}$.

The hydrogen caused an immediate but temporary improvement in the conversion rate. Within a few hours, after most of the hydrogen gas was consumed, conversion gradually reverted to the preinjection condition. We believe the temporary improvement is primarily a temperature effect, since the heat of reaction is sufficient to cause a temperature $\mathrm{rise}$ of about $10 \mathrm{~K}$ at the initial hydrogen concentration.

Ostlund ${ }^{8}$ found that his catalyst performance became unreliable without the continual addition of about $0.3 \% \pm 0.1 \%$ by volume of hydrogen carrier gas. He thought that there was some inherent catalytic limitation unless the total hydrogen level was well above ambient. We do not find this to be true in our experiments, which generally start at the ambient normal hydrogen level (excepting the test just described), and then drop below that level by several orders of magnitude. We believe the poor performance of ostlund's ambienttemperature reactor can be explained primarily by inadequate gas-residence time. We would, of course, expect the temperature increase from hydrogen addition to be beneficial through the customary mechanism of increasing the rate coefficient. 
We next tried to reactivate the catalyst in situ by continuous hydrogen injection. This was done without tritium in the enclosure, with the stack vent open and the enclosure door ajar. We bled a stream of hydrogen gas into the processing loop at $0.2 \mathrm{l} / \mathrm{min}$, and controlled the total air flow at $20 \mathrm{l} / \mathrm{min}$. This produced a 18 hydrogen concentration which was maintained for about $3 \mathrm{~h}$. The catalyst-bed outlet pipe became noticeably, warm to the touch from the heat of reaction.

After cooling overnight, we carried out the tritilum conversion run shown in Fig. 14. The conversion slope is the same as before the hydrogen treatments discussed above, and we conclude that these mild treatments are inadequate for reactivation. The data for this run, summarized in Table 4, show that the rate coefficient at 336 days after air exposure has decreased to $0.205 \mathrm{~s}^{-1}$, about an order of magnitude smaller than a: 0.1 days.

The tritium concentration in Fig. 14 begins to level out at about 1 day, and by 2 days bases out at $2200 \mu \mathrm{Ci} / \mathrm{m}^{3}$. This rather elevated baseline is caused by the gradual growth of tritiated methane in the tritium injector. We did not correct the conversion-slope estimate for this residual tritium level. Had we done so, the estimated rate coefficient would increase about 58 .

Pt/ALUMINA (AGE $=380 \mathrm{~d})$

The Pt/alumina catalyst was rerun after more than one year of exposure to air, and the results are shown in Fig. 15. The rapid initial drop in concentration from mixing after tritium injection, evident in Figs. 10 through 14, does not appear in Fig. 15, since for this run our zero time does not correspond to tritium injection time. We do not show the first portion of the run during which we sampled the gas in the tritium injector for chromatographic analysis, removed the sample vessel from the enclosure, and then allowed about $15 \mathrm{~min}$ for equilibration prior to recording data.

Figure 15 shows conversion data to $10 \mathrm{~h}$. There is little reaction at later times, although we continued the experiment for several days to get a better estimate of the final residual concentration-about $2450 \mathrm{\mu Ci} / \mathrm{m}^{3}$. The data shown in Fig. 15 have been corrected for thils residual concentration, and we have included approximate error bars to show the large uncertainty in late-time data as the residual baseline is approachecl. 


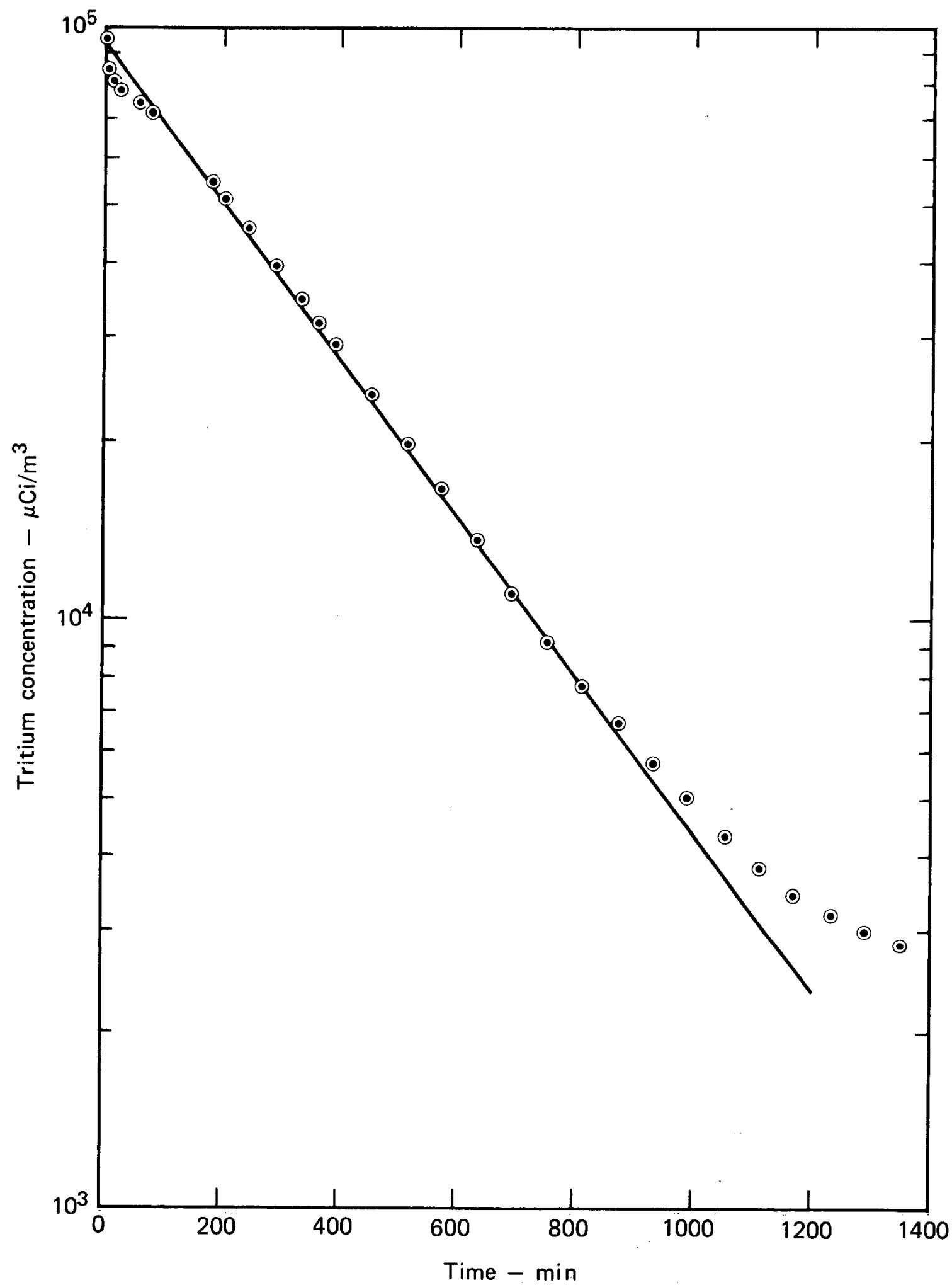

FIG. 14. Tritium/air catalytic oxidation with Pd/kaolin (age = 336 d). Data shown for dry ion chamber. Conversion slope $=0.00306 \mathrm{~min}^{-1}$. Fractional conversion $=0.110$. Rate coefficient $=0.205 \mathrm{~s}^{-1}$. 


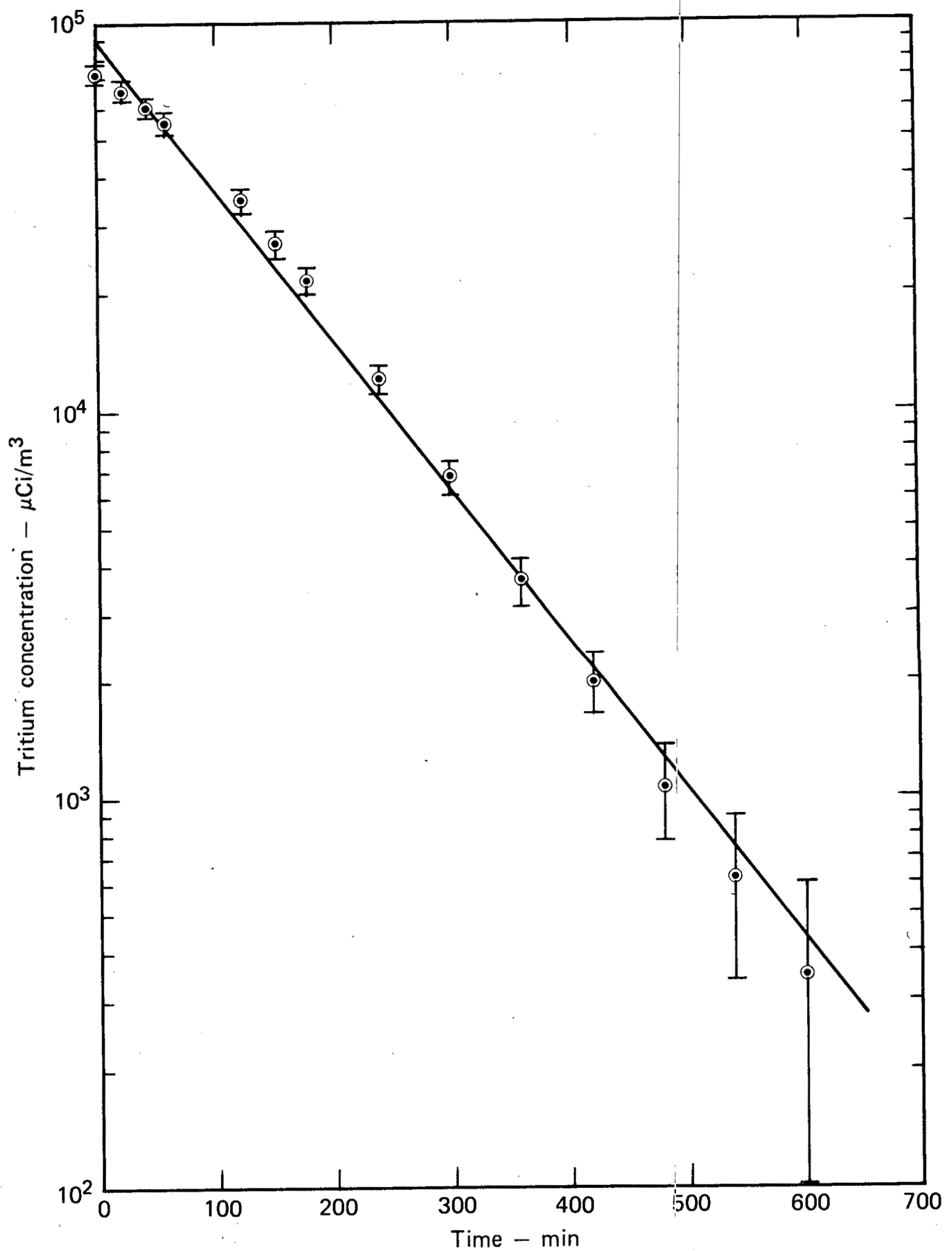

FIG. 15. Tritium/air catalytic oxidation with Pt/alunina (age $=380 \mathrm{~d}$ ). Data shown for $\mathrm{dry}$ ion chamber. Conversion slope $=0.00890 \mathrm{~min}^{-1}$. Fractional conversion $=0.321$. Rate coefficient $=0.645 \mathrm{~s}^{-1}$. Data have been corrected for a residual baseline concentration of $2450 \mu \mathrm{Ci} / \mathrm{m}^{3}$. The error bars show increasing uncertainty in late-time data. 
The rate coefficient, based on the calculations summarized in Table 4, has decreased about an order of magnitude at 380 days to $0.645 \mathrm{~s}^{-1}$.

$\mathrm{Pd} / \mathrm{ZEOLITE}(\mathrm{AGE}=1 \mathrm{~d})$

Test results for the $\mathrm{Pd} / z e o l i t e$ catalyst prepared by the method described earlier, then activated and aged 1 day in air, are shown in Fig. 16. Beginning with this $r$ un, we adopted a different procedure for test initiation. Tritium is injected and allowed to mix in the enclosure and circulate through the processing loop. During the mixing period, the catalyst bed is bypassed. After the tritium concentration has leveled out, the catalyst bed is brought on-line for the conversion test.

Figure 16 shows that mixing time is about $10 \mathrm{~min}$, consistent with the earlier data shown in Fig. 10 for an unactivated catalyst. Tritium concentration then remains constant until the catalyst is brought on-line, at which time conversion begins to reduce the concentration rapidly. An instrument scale change is noticeable in Fig. 16 at about $70 \mathrm{~min}$, where we used two displaced lines with the same slope, a procedure that was also followed in Fig. 12 .

As a further verification that the straight-line conversion slope is attributable to the catalyst, we valved the catalyst bed off-line at $108 \mathrm{~min}$ and then resumed conversion at $158 \mathrm{~min}$. The response shown in Fig. 16 provides the desired verification: a straight-line downslope followed by a horizontal plateau, and then a resumption of conversion with the same downslope.

The data shown in Fig. 16 are from the moist-air ion chamber, and have been corrected for a residual concentration of $6180 \mu \mathrm{Ci} / \mathrm{m}^{3}$. Test results, as summarized in Table 4 , show a 95.68 conversion in the cataly tic reactor and a rate coefficient of $14.9 \mathrm{~s}^{-1}$.

RESIDUAL TRITIATED METHANE

Chromatographic analysis of a sample drawn from the tritium-injector vessel prior to the $\mathrm{Pd} / \mathrm{zeolite}$ test showed a methane content of $103 \mathrm{ppm}$. This figure should be quite accurate since a methane standard of nearly the same concentration was used in the analysis to calibrate the peak response.

Morris ${ }^{12}$ studied methane formation in stainless steel vessels containing tritium. He based his results on exposed-metal surface area, and reported 


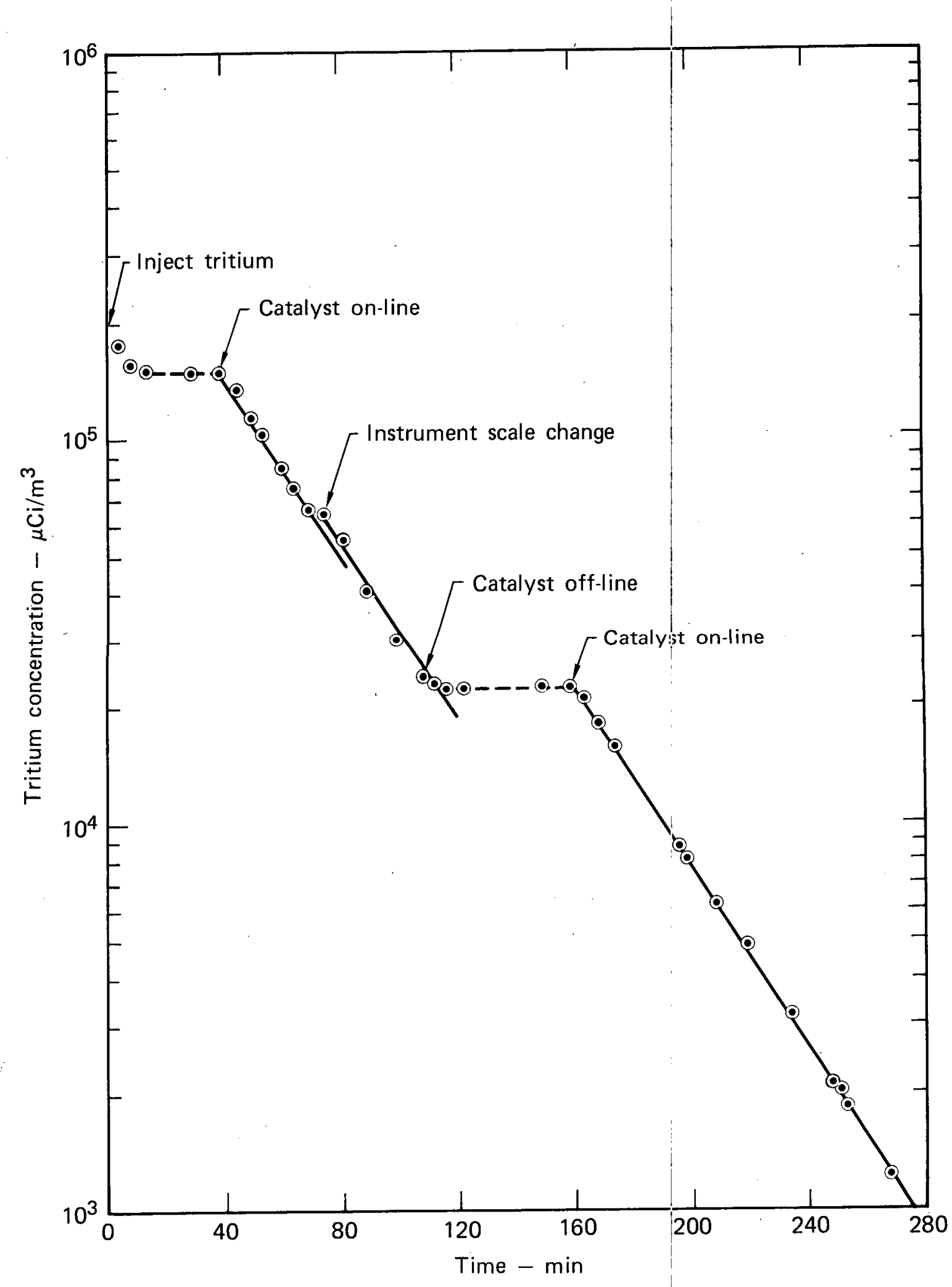

FIG. 16. Tritium/air catalytic oxidation with $\mathrm{Pt} /$ zecilite (age $=1$ d). Data shown for moist-air ion chamber. Conversion slope $=0.0265 \mathrm{~min}^{-1}$. Fractional conversion $=0.956$. Rate coefficient $=14.9 \mathrm{~s}^{-1}$. Dáta have been corrected for a residual baseline concentration of $6180 \mu \mathrm{Ci} / \mathrm{m}^{3}$. 
methane content at equilibrium of approximately $10 \mathrm{~cm}^{3}$ (STP) $/ \mathrm{m}^{2}$, with a factor-of-three variation depending on surface treatment. Our methane content of $103 \mathrm{ppm}$ is equivalent to $23 \mathrm{~cm}^{3}$ (STP) $/ \mathrm{m}^{2}$, based on the known injectorvessel volume, pressure, and a surface area estimated from the vessel dimensions. This number is in good agreement with the study by Morris.

The chromatograph does not tell us the isotopic composition of the methane. Tritiated methane will probably predominate, since about $93 \%$ of the isotopic hydrogen atoms introduced originally into the vessel were tritium atoms. Assuming the same isotopic proportions for methane and allowing for tritium decay, we calculate that the tritiated methane activity is $2.4 \%$ of the total tritium activity in the injector vessel at the time of the chromatographic analysis.

We can compare our estimate of tritiated methane activity with ion-chamber measurements made during the Pd/zeolite test, which took place a few days after the chromatographic analysis. To make the comparison we assume that the residual concentration measurements at the end of the test run represent tritiated methane and that the initial measurements, after injection and mixing but before catalytic conversion, represent total tritium activity. The results, given in Table 5, show 3.0 and $4.0 \%$ residual activity as measured by the two ionization chambers. The difference does not represent tritiated water since we purposely bypassed the adsorbent bed and directed moist air to the chamber that normally receives dry air. The chromatographic analysis thus "explains" 60-80\% of the residual activity as tritiated methane, depending on which (if either) of the two sets of ion chamber measurements you prefer to believe.

The enclosure air contacts oil-wet seals as it passes through the recirculation pump. In view of this fact, together with the disagreement between the two chambers, we feel that the residual activity is substantially all tritiated hydrocarbons, mainly methane, perhaps mixed with some higher molecular weight pump-oil volatiles.

Bixel and Kershner ${ }^{7}$ also found that a trace of tritiated hydrocarbons in their source gas could not be oxidized with an ambient temperature catalyst. Their mass-spectrometric analysis was unable to identify the offending species. We have belabored this question in order to try and pin down the chemical species, and also to make it less plausible that a change in mechanism might stop hydrogen oxidation at lower concentrations. 
TABLE 5. Comparison of initial and residual tritium concentrations during Pd/zeolite (age $=1$ d) catalyst test. ${ }^{a}$

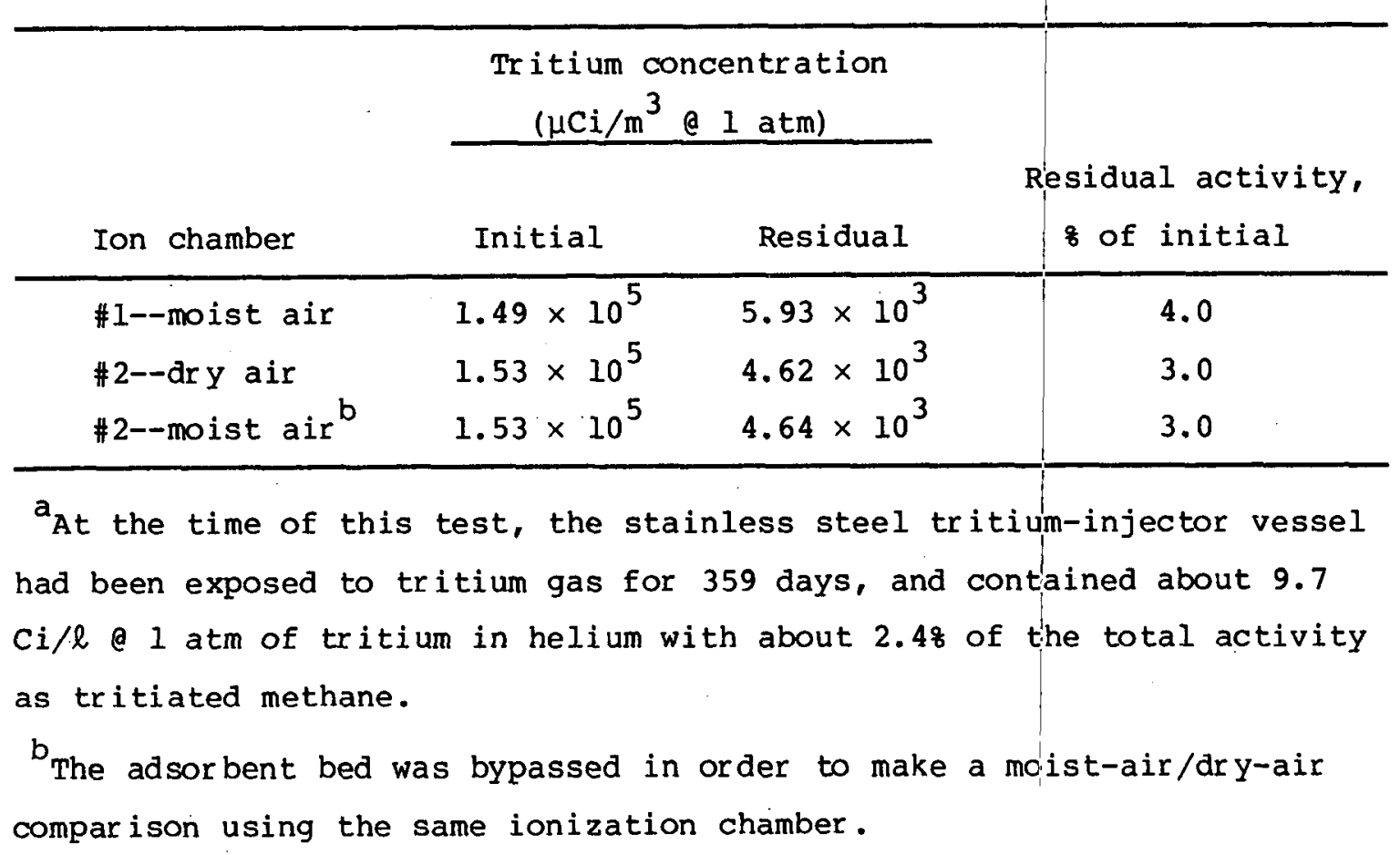

$\mathrm{Pd} / \mathrm{ZEOLITE}(\mathrm{AGE}=35 \mathrm{~d})$

The Pd/zeolite catalyst was retested at 35 days cif air exposure and the results are shown in Fig. 17. In most respects this test is quite similar to the earlier test shown in Fig. 16 except that catalyst performance has degraded somewhat. Table 4 shows that conversion has dropped to $42.8 \%$ and the rate coefficient is down to $2.73 \mathrm{~s}^{-1}$.

Pd/KAOLIN (REACTIVATED, AGE $=2$ d)

In view of our unsuccessful attempt to reactivate the Pd/kaolin catalyst with a mild hydrogen treatment, we removed the catalyst from the processing$100 \mathrm{p}$ canister and subjected it to the standard hot-hydrogen treatment discussed in an earlier section of this report.

Test results after 2 days of exposure to air are|shown in Fig. 18, where data are presented for the moist-air ion chamber. The reader may wonder why 


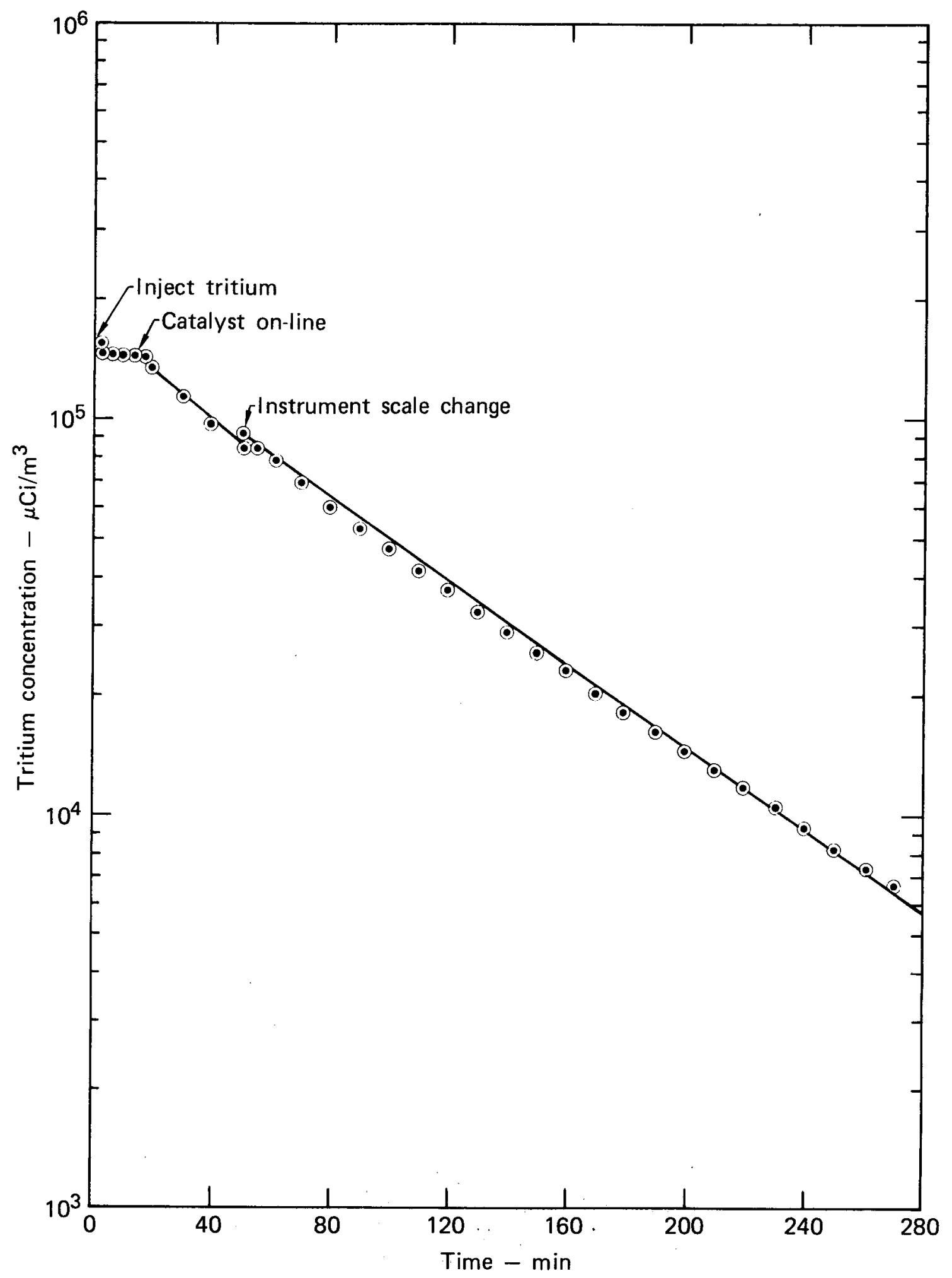

FIG. 17. Tritium/air catalytic oxidation with Pd/zeolite (age $=35$ d). Data shown for moist-air ion chamber. Conversion slope $=0.0121 \mathrm{~min}^{-1}$. Fractional conversion $=0.428$. Rate coefficient $=2.73 \mathrm{~s}^{-1}$. Data have been corrected for a residual baseline concentration of $6600 \mu \mathrm{Ci} / \mathrm{m}^{3}$. 


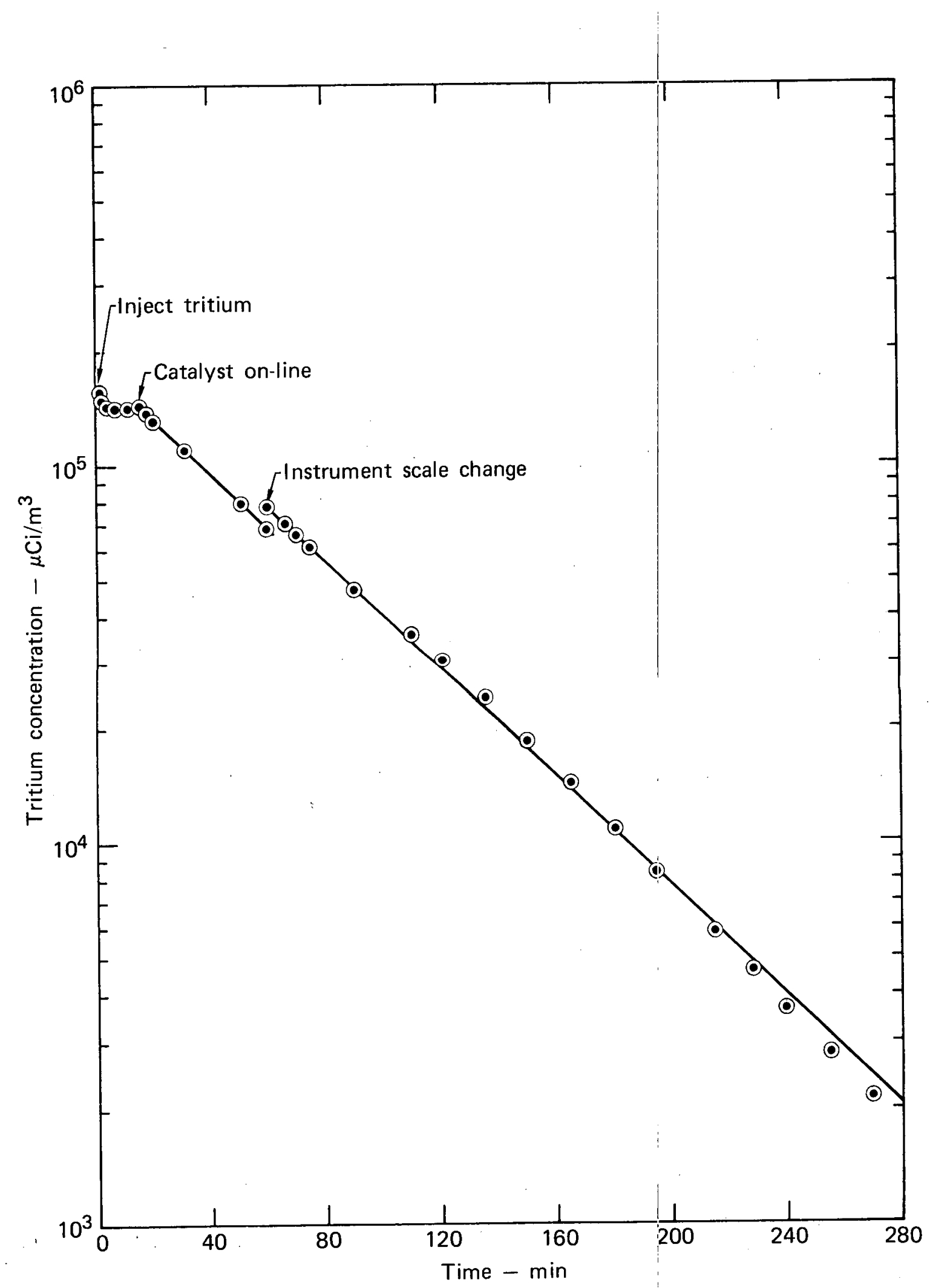

FIG. 18. Tritium/air catalytic oxidation with Pd/kaolin (reactivated age $=2 \mathrm{~d}$ ). Data shown for moist-air ion chamber. Conversion slope $=0.0165 \mathrm{~min}^{-1}$. Fractional conversion $=0.573$. Rate coefficient $=1.59 \mathrm{~s}^{-1}$. Data have been corrected for a residual baseline concentration of $7090 \mu \mathrm{Ci} / \mathrm{m}^{3}$. 
we are not using the dry-air chamber data in order to detect changes in tritium gas concentration. The answer is twofold: the dry-air chamber has become unreliable, and there appears to be very little tritiated water present anyway, as indicated in Table 5. The defect in the dry-air chamber that appeared during the last two test runs is an inability to up-range beyond $10^{5} \mu \mathrm{Ci} / \mathrm{m}^{3}$. When readings fall below that level the chamber appears to read properly down to $10^{3} \mu \mathrm{Ci} / \mathrm{m}^{3}$. At lower levels, the reading drops abruptly to zero. The slope of the line in Fig. 18 corresponds to 57.38 conversion, and with the other data given in Table 4 we calculate a rate coefficient of $1.59 \mathrm{~s}^{-1}$. A comparison with the $1.75 \mathrm{~s}^{-1}$ initial value in Table 4 shows that we have effectively restored the degraded catalyst to its prior state of high activity. 
All of the kinetic rate coefficient data from our experimental runs are presented in Fig. 19 as a function of exposure time to air after activation. It is interesting to observe that the decline of catalyst activity with age suggests a power law with an exponent of $-1 / 2$. We have no theory to explain this behavior, however, and there are few data for each catalyst. But it does seem clear, for the three catalysts tested, that catalytic activity degrades steadily with air-exposure time, showing an order of magnitude drop after about one year.

It is tempting to speculate that oxygen is the culprit behind the catalyst degradation. A trace amount of some unknown contamincint in the air might seem a plausible suspect had we circulated air through the catalyst bed between tests. Instead, the catalyst canister was sealed. But there is ample oxygen in the canister volume to oxidize completely the small. amount of catalyst present. As indirect evidence, we know that another on-line catalyst in our facility has functioned for years in an argon atmosphere. The evidence is only qualitative, however, and some degradation may in fact have occurred in that catalyst, which incidentally runs at an elevated temperature.

At the same catalyst age, the Pd/zeolite catalys: has the highest rate coefficient, followed by the Pt/alumina catalyst, with the Pd/kaolin catalyst showing the lowest catalytic activity. This ranking of effectiveness is not unexpected; it follows the precious metal content lis ted in Table 2.

A more enlightening basis for comparison of the three catalyst's is by means of the surface rate coefficient. So far, we have extracted the rate coefficient defined by Eq. (4) from the experimental data. This is the pseudohomogeneous (volume) coefficient, with units of reciprocal time, representing the volume of gas reacting per unit time/per unit volume of catalyst bed. In fact, we know that the oxidation reaction is primarily heterogeneous and surface-catalyzed. The equation relating the two coefficients may be written:

$$
\mathrm{K}=\eta \mathrm{kS} \rho_{b^{\prime}}
$$

where $k$ is the volume rate coefficient, $k$ is the surface rate coefficient, $s$ is the dispersed-metal catalyst surface area per unit pellet mass, $\rho_{b}$ is the pellet-bed bulk density, and $n$ is the catalyst effectiveness factor. 6 


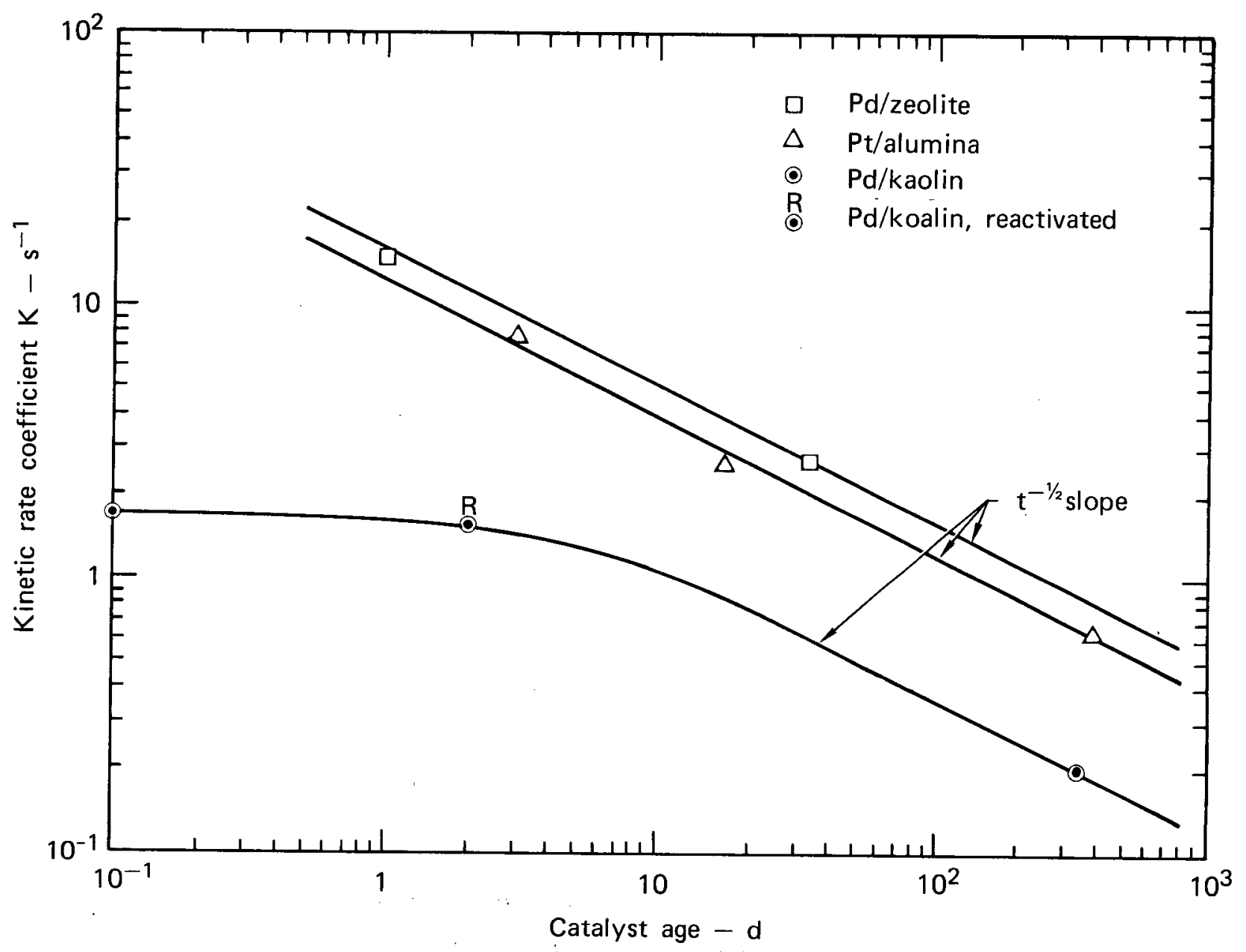

FIG. 19. Kinetic rate coefficient: for tritium/air catalytic oxidation at ambient temperature $(24-27$ c) as a function of time exposed to air after activation. 
The effectiveness factor is a correction for the rate-limiting effects of diffusion within the pellet and boundary-layer mass transfer resistance at the pellet surface. We have made rough calculations which indicate that these rate-limiting corrections are small for our ambient-temperature experiments, in which case $\eta \simeq 1$. With this simplification, we rearrange Eq. (5) to calculate the surface rate coefficient:

$$
k=k /\left(S \rho_{b}\right)
$$

The surface coefficient $k$ has units of velocity and represents the volume of gas reacting per unit time per unit area of catalyst surface. It is not a simple matter to choose the proper surface area. For some reactions the entire pellet area might be effective, in which case we would use the B.E.T. area given in Table 1. For this study we will use the dispersed-metal particle area estimated in Table 2.

A comparison of surface rate constants, calculated from Eq. (6), is shown in Table 6 at the same catalyst age $(100 \mathrm{~d})$ for the three catalysts. It can be seen that the $\mathrm{Pt} /$ alumina catalyst is the most effective on a surfacecoefficient basis, and that the two palladium-based catalysts are about equal and lower by a factor of 3 .

Bixel and Kershner ${ }^{7}$ obtained a measurement at $23 \mathrm{C}$ on the same type of pt/alumina catalyst that we tested. Their rate value of $1.4 \mathrm{~s}^{-1}$ shown in Table 6 agrees well with our $1.2 \mathrm{~s}^{-1}$ number at 100 days age. This agreement is important for two reasons. First, it lends confidence to our experimental measurement program insofar as two sets of investigators are in substantial agreement using the same catalytic material. Second, it is reassuring to confirm the earlier measurement that has been used as the basis for design studies of tritium cleanup systems for fusion reactor/s. ${ }^{13}$ Bixel and Kershner also measured the oxidation rate at $177 \mathrm{C}$, where the rate coefficient was higher by a factor of 60 . To our knowledge, the two data points of these investigators and the single datum on platinum foil discussed below constitute the only documented rate coefficients for tritium/air catalytic oxidation in the literature prior to this study.

Kulacki and Gidaspow ${ }^{14}$ measured hydrogen oxidation, at a concentration of about 18 in air, on a platinized-alumina slab in the temperature $r$ ange 70-330 C. Their method of alumina-impregnation is analogous to our pellet 
TABLE 6. Tritium/air oxidation rate coefficients at ambient temperature (K denotes pseudohomogeneous reaction; $k$ denotes heterogeneous surface reaction).

\begin{tabular}{|c|c|c|c|c|c|}
\hline \multirow[b]{2}{*}{ Catalytic material } & \multicolumn{3}{|c|}{ Concentration } & \multirow[b]{2}{*}{$\begin{array}{c}k \\
(\mu \mathrm{m} / \mathrm{s})\end{array}$} & \multirow[b]{2}{*}{ Reference } \\
\hline & $\begin{array}{l}\text { Reaction } \\
\text { order }\end{array}$ & $\begin{array}{r}r \text { ange } \\
\left(\mu \mathrm{Ci} / \mathrm{m}^{3}\right)\end{array}$ & $\left(s^{-1}\right)$ & & \\
\hline Pt/alumina pellets ${ }^{a}$ & 1 & $10^{2}-10^{5}$ & 1.2 & 2.1 & This work \\
\hline $\mathrm{Pd} /$ kaolin pellets ${ }^{\mathrm{a}}$ & 1 & $10-10^{5}$ & 0.37 & 0.76 & This work \\
\hline Pd/zeolite pellets ${ }^{a}$ & 1 & $10^{4}-10^{5}$ & 1.6 & 0.67 & This work \\
\hline Pt/alumina pellets & 1 & $10^{2}-10^{4}$ & 1.4 & - & Ref. 7 \\
\hline Platinized-alumina slab ${ }^{b}$ & 1 & $\left(10^{10}\right)^{c}$ & - & 0.15 & Ref. 14 \\
\hline Platinum foil ${ }^{\mathrm{d}}$ & 2 & $2 \times 10^{7}$ & $4.7 \times 10^{-6}$ & 0.13 & Ref. 15 \\
\hline None $e^{d}$ & 2 & $2 \times 10^{7}$ & $1.6 \times 10^{-8}$ & - & Ref. 15 \\
\hline None ${ }^{d}$ & $5 / 3$ & $10^{6}-10^{12}$ & $1.2 \times 10^{-9}$ & - & Ref. 17 \\
\hline
\end{tabular}

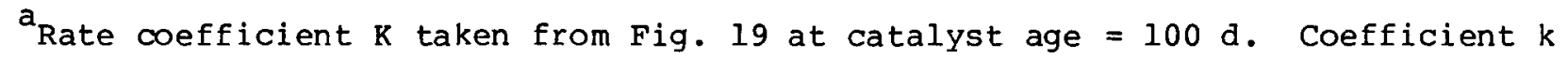
calculated from $\mathrm{K}$ using Eq. (6).

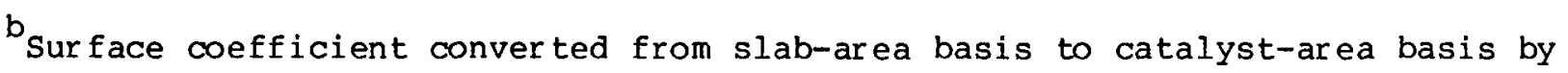
method given in Table 2, footnote d. Rate coefficient extrapolated to $25 \mathrm{C}$ from data in the range $70-330 \mathrm{C}$.

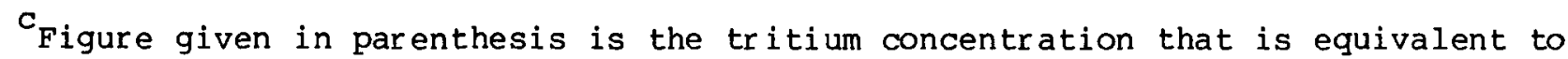
the hydrogen concentration used in experiment.

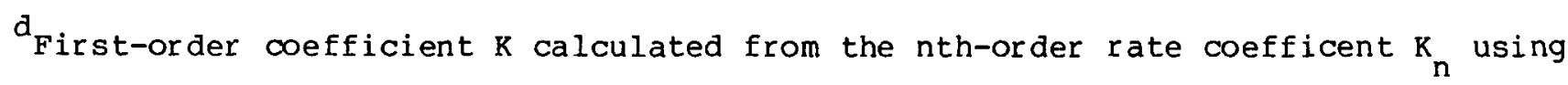
$\mathrm{K}=\mathrm{K}_{\mathrm{n}} \mathrm{C}_{0}^{\mathrm{n}-1}$, with $\mathrm{C}_{0}=2 \times 10^{7} \mu \mathrm{Ci} / \mathrm{m}^{3}$.

preparation method in that they also applied a metal chloride solution. Their deposited particles may be about the same size as we used in our surface-area calculation for Table 2. Assuming this to be the case, we calculate a surface rate coefficient of $0: 15 \mathrm{~s}^{-1}$, as shown in Table 6 . We consider the agreement with our peliet measurements to be satisfactory in view of the extrapolation from their higher-temperature data. 
Eakins and Hutchinson ${ }^{15}$ measured tritium/air oxidation within a glass bulb in the presence of a platinum metal foil. They evaluated a rate coefficient based on a second-order reaction. The assumed reaction order is conjectural since the concentration range covered in their experiments is small. Converting their data to a first-order basis gives the volume and surface rate coefficients shown in Table 6 . It can be seen that the surface coefficient for platinum foil is comparable to those shown for the dispersedmetal catalysts. The great disparity in volume coefficients is evidently just a matter of surface area; tiny particles are much more effective than metal foil.

In the last two rows of Table 6 we show, for comparison, estimated volume rate coefficients for tritium/air oxidation in the absence of a catalyst. Scanning the column of numbers shows an impressive spread of 9 orders of magnitude between rate coefficients for dispersed-metal catalyst and no catalyst at all. From this perspective, the differences in rate coefficients between the three dispersed-metal catalysts under investigation is almost trivial. All three are remarkably active.

Table 6 also summarizes the tritium concentration range covered during experiments with the three catalysts. The widest range of catalytic reaction, from about 10 to $10^{5} \mu \mathrm{Ci} / \mathrm{m}^{3}$, has been measured with the $\mathrm{Pd} / \mathrm{kaolin}$ catalyst. We observe first-order kinetics over this 4-order-of-magnitude concentration range. Bixel and Kershner's data cover the range $10^{2}$ to $10^{4} \mu \mathrm{Ci} / \mathrm{m}^{3}$ and we have therefore extended their region of observed first-order catalytic behavior by about 1 order of magnitude in each direction.

Although no hydrogen carrier gas was used in our experiments, we started each experiment with ambient-air hydrogen concentration of about $0.5 \mathrm{ppm}$. The hydrogen isotope abundance exceeded that of the tritium isotope by a factor ranging from about 10 to 50 at the start of an experimental $r$ un. The hydrogen isotope may react somewhat faster than the heavier tritium isotope, ${ }^{16}$ but in first approximation we can neglect the kinetic isotope effect. The maximum tritium-concentration reduction obtained in a single/experiment is about 3 orders of magnitude. Therefore, another way to phrase our kinetic results is to assert that we have measured tritium-traced hydrogen oxidation, and that we observed first-order kinetics over a 3-order-of-magnitude concentration range for hydrogen, from 0.5 parts per million down to 0.5 parts per billion. 
We now consider some engineering aspects of the catalyst kinetic data and in particular the problem engendered by catalyst aging. There may be special circumstances where aging can be avoided. For example, if a system is used only infrequently it might be possible to blanket the standby catalyst with inert gas to prevent degradation. We will assume this option is unavailable.

DECONTAMINATION FACTOR

A design specification for tritium/air cleanup systems is often stated in terms of a decontamination factor. We assume for simplicity that the system consists of a catalytic converter followed by an adsorbent bed which captures all the entering water vapor. The decontamination factor, $D$, is then related simply to the fractional conversion, $f$, of tritium gas in the catalyst bed:

$$
D=1 /(1-f) \text {. }
$$

The catalyst volume required to achieve a specified decontamination factor follows from Eqs. (4) and (7):

$$
V_{C}=Q \operatorname{lnD} / K \text {. }
$$

Unfortunately, the rate coefficient $\mathrm{K}$ slowly declines with catalyst age as shown in Fig. 19. Assuming a $t^{-1 / 2}$ age dependence,

$$
K=K_{0}\left(t / t_{0}\right)^{-1 / 2}
$$

where $K_{0}$ is the rate coefficient corresponding to some reference age $t_{0} \cdot$ The catalyst volume, from Eqs. (8) and (9), is then

$$
v_{c}=\left(t / t_{0}\right)^{1 / 2} Q \operatorname{lnD} / K_{0}
$$

If we choose the system catalyst volume using Eq. (10) with time $t=t_{0}$, then as the catalyst ages the decontamination factor must diminish from the initial value, $D_{0}$. From Eq. (10) it follows that

$$
D=D_{0} \sqrt{t_{0} / t},
$$

where

$$
D_{0}=\exp \left(V_{c} K_{0} / Q\right) .
$$


Note that $D_{0}$ in Eq. (11) is raised to a power which diminishes with increasing time, and therefore $D$ will eventually approach unity where the catalytic converter is totally ineffective. Again, we emphasize that the dispersed-metal catalysts are very, active, and large initial decontamination factors (Eq. (12)) may often be obtained with a reasonable volume of catalyst. The behavior of the decontamination factor with catalyst age is shown in Fig. 20. The asymptotic approach to unity can be delayed by starting with a larger catalyst volume. If the minimum acceptable decontamination factor is 10, for example, then from Fig. 20 reactivation must occur at either 4, 36, or 400 days for relative catalyst volumes of 1, 3, or 10, respectively. Clearly there will be an optimum reactivation time which will depend, among other things, on catalyst and activation costs. To gain a better understanding of the factors involved, we next explore an optimization procedure for a simplified scenario.

OPTIMUM REACTIVATION TIME

Let $\Delta$ represent the reactivation cycle time, as sketched in Fig. 21 . Assume for simplicity that activation time is negligilble compared to the on-stream part of the cycle, and that the processing isystem can be shut down briefly during reactivation. Otherwise we would requile two processing units in parallel, one on-stream and one being reactivated.

Let $c_{C}$ represent the cost of catalyst per unit volume, and let $c_{r}$ represent the cost per reactivation. Reactivation cost is probably related to catalyst volume, especially when comparing very large and very small systems, but we will neglect this dependence and assume that $c_{r}$ is essentially a fixed man-hour charge. The total cost, $c_{T}$, which we want to minimize, is the sum of catalyst cost plus all future reactivation charges discounted back to the present:

$$
\mathrm{c}_{\mathrm{T}}=\mathrm{c}_{\mathrm{c}} \mathrm{v}_{\mathrm{c}}+\sum_{\mathrm{n}} \mathrm{c}_{\mathrm{r}} \exp (-1 \mathrm{n} \Delta) \text {. }
$$

In Eq. (13) the exponential discount factor's correspond to multiples of the cycle time, $n \Delta$, and correct future costs back to the present using continuous discounting with an annual interest rate.$_{+}{ }^{*}$ The summation

\footnotetext{
*For those who prefer to compound interest $q$ times per year at annual rate $i$ ', the equivalent continuous rate is$$
i=q \ln (1+i / q) \text {. }
$$ 


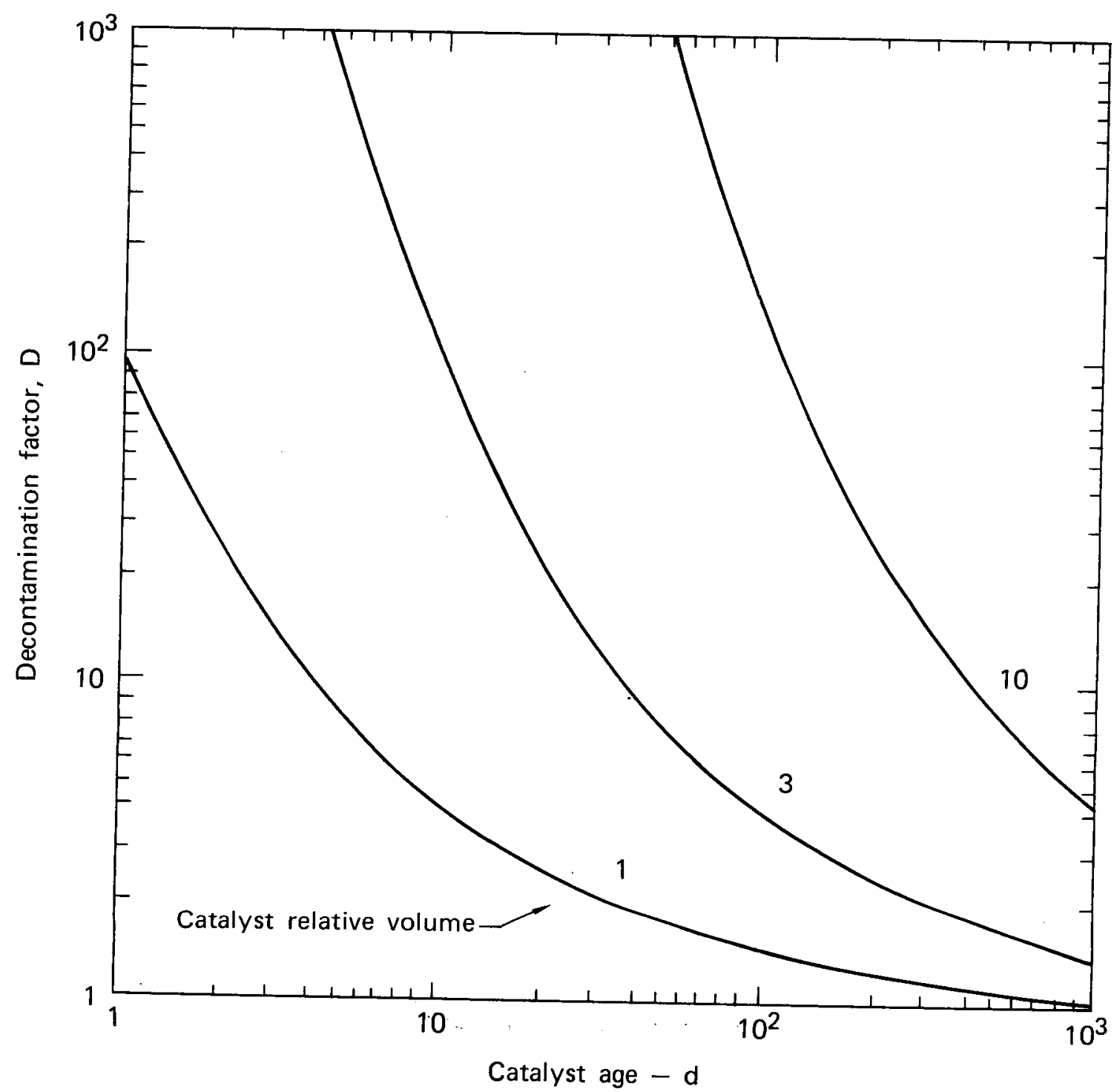

FIG. 20. Cleanup system decontamination factor as a function of catalyst age, based on $t_{0}=1$ day and $D_{0}=10^{2}$. The asymptotic approach to unity can be delayed if a larger volume of catalyst is used. 


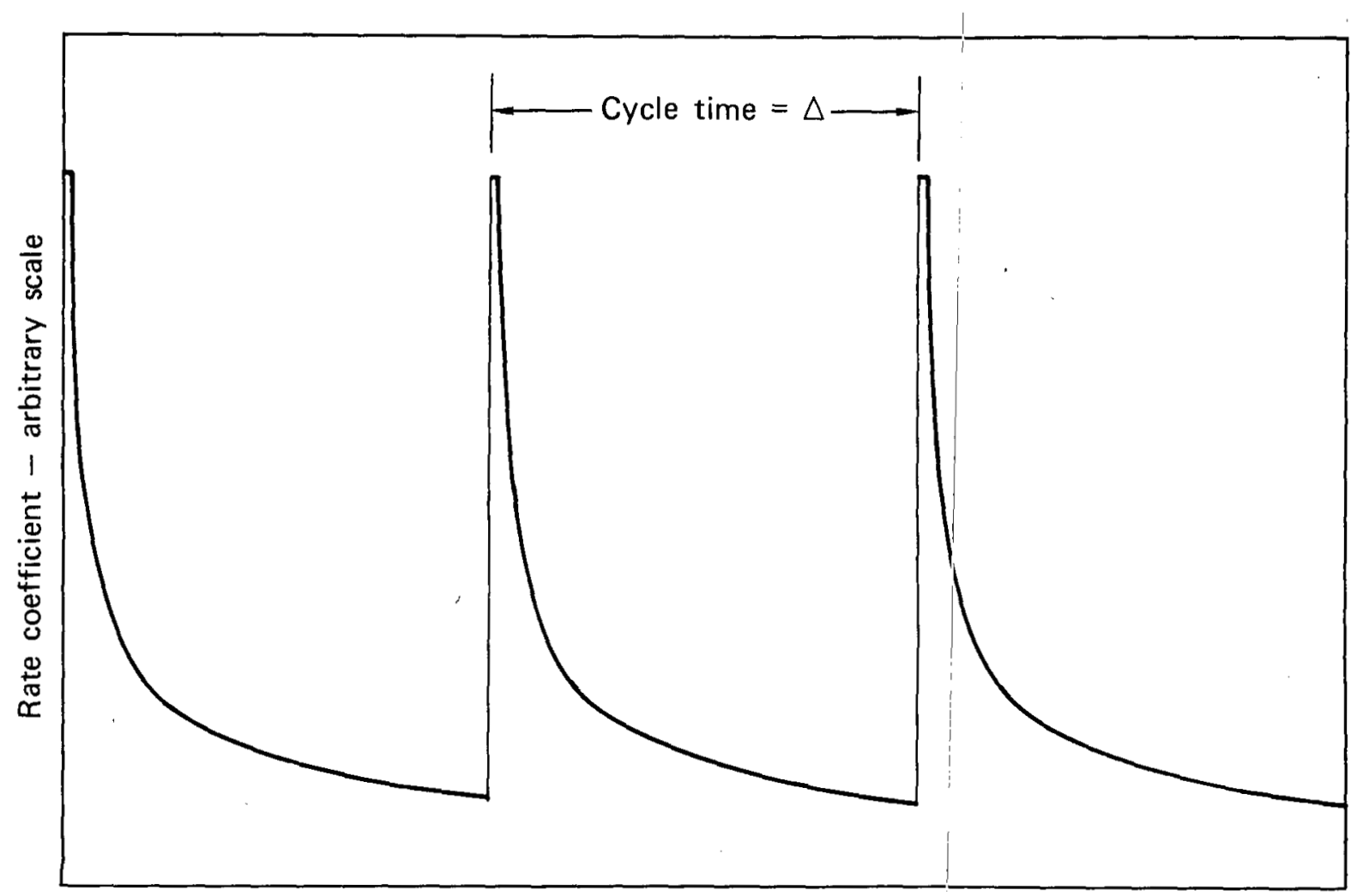

Time - arbitrary scale

FIG. 21. Catalyst rate coefficient as a function of time, with cycle-time $\Delta$ between reactivations.

Is a geometric progression and, assuming we have a large number of terms, Eq. (13) may be written

$$
c_{T}=c_{C} v_{c}+c_{r} /[1-\exp (-i \Delta)] \text {. }
$$

Substituting for the catalyst volume from Eq. (10),

$$
c_{T}=c_{C}\left(\Delta / t_{0}\right)^{1 / 2} Q \operatorname{lnD} / K_{0}+c_{r} /[1-\exp (-i \Delta)] \text {. }
$$

To find the optimum (minimum cost) cycle time, we differentiate Eq. (15) with respect to $\Delta$, set the derivative equal to zero, and solve the resulting equation for cycle time. For i $\Delta<1$, an excellent approximate solution is

$$
\Delta=\left[2\left(c_{r} / c_{c}\right)\left(t_{0}\right) 1 / 2 k_{0} /(i Q \ln D)\right]^{2 / 3} \text {. }
$$

Substituting this cycle time into Eq. (10) for catalyst volume gives

$$
\left(\mathrm{V}_{\mathrm{c}}\right)_{\text {optimum }}=\left[2 \mathrm{c}_{\mathrm{r}} /\left(\mathrm{c}_{\mathrm{c}} 1 \mathrm{t}_{0}\right)\right]^{1 / 3}\left(\mathrm{Q} \operatorname{lnD} / \mathrm{K}_{0}\right)^{2 / 3} \text {. }
$$


Finally, the minimum total cost turns out to be

$$
\left(c_{T}\right)_{\text {minimum }}=\frac{3}{2} c_{C}\left(v_{C}\right)_{\text {optimum }},
$$

i.e., at minimum cost the discounted value of future activation costs equals 508 of the initial catalyst cost.

We note that the optimum catalyst volume of Eq. (17) scales with flow rate raised to the $2 / 3$ power, in contrast to the customary direct proportionality implied by Eq. (8) for an age-independent catalyst. Also, it is comforting to observe that catalyst volume and cost (which is proportional to volume) are relatively insensitive to uncertainties in the reactivation cost because of the $1 / 3$ power scaling.

Table 7 shows the variation with flow rate of optimum cycle time, and optimum catalyst volume and associated catalyst cost, based on parameter values listed with the table. We show a range of flow rate varying from a small-scale cleanup system to a very large system appropriate to fusion reactor-hall service. Frequent reactivation becomes attractlve at high flow rate due to the large investment in catalyst. At very low flow rate, reactivation is virtually unnecessary since catalyst cost is insignificant. We belleve that the trend toward shorter cycle time at high flow rate is correct, while granting that our quantitative estimate will be altered by a different choice of parameter values.

A system designer will want to consider heating the catalyst to increase the rate constant and thereby reduce the volume requirement. If a heated catalyst is used, the air flow leaving the converter will need to be cooled to near-ambient temperature in order to prevent degradation of adsorbent-bed performance. The cost of heat exchange (both heating and cooling) will need to be balanced against potentlal catalyst cost savings. Heat-exchange cost scales approximately with flow rate to the $1 / 2$ power, and will not be attractive at low flow rate where catalyst cost is minor. We have made rough cost estimates which indicate that heat-exchange cost is about in balance with catalyst savings in the 0.1 to $1 \mathrm{~m}^{3} / \mathrm{s}$ flow rate range. For the large $10 \mathrm{~m}^{3} / \mathrm{s}$ system shown in Table 7, it will prove economic to use a heated catalyst. An approximate indication of total system cost can be obtalned from Table 7 by scaling up the catalyst cost by an order of magnitude. 
TABLE 7. Catalyst cost as a function of cleanup system flow rate, based on optimum cycle time and catalyst volume. al

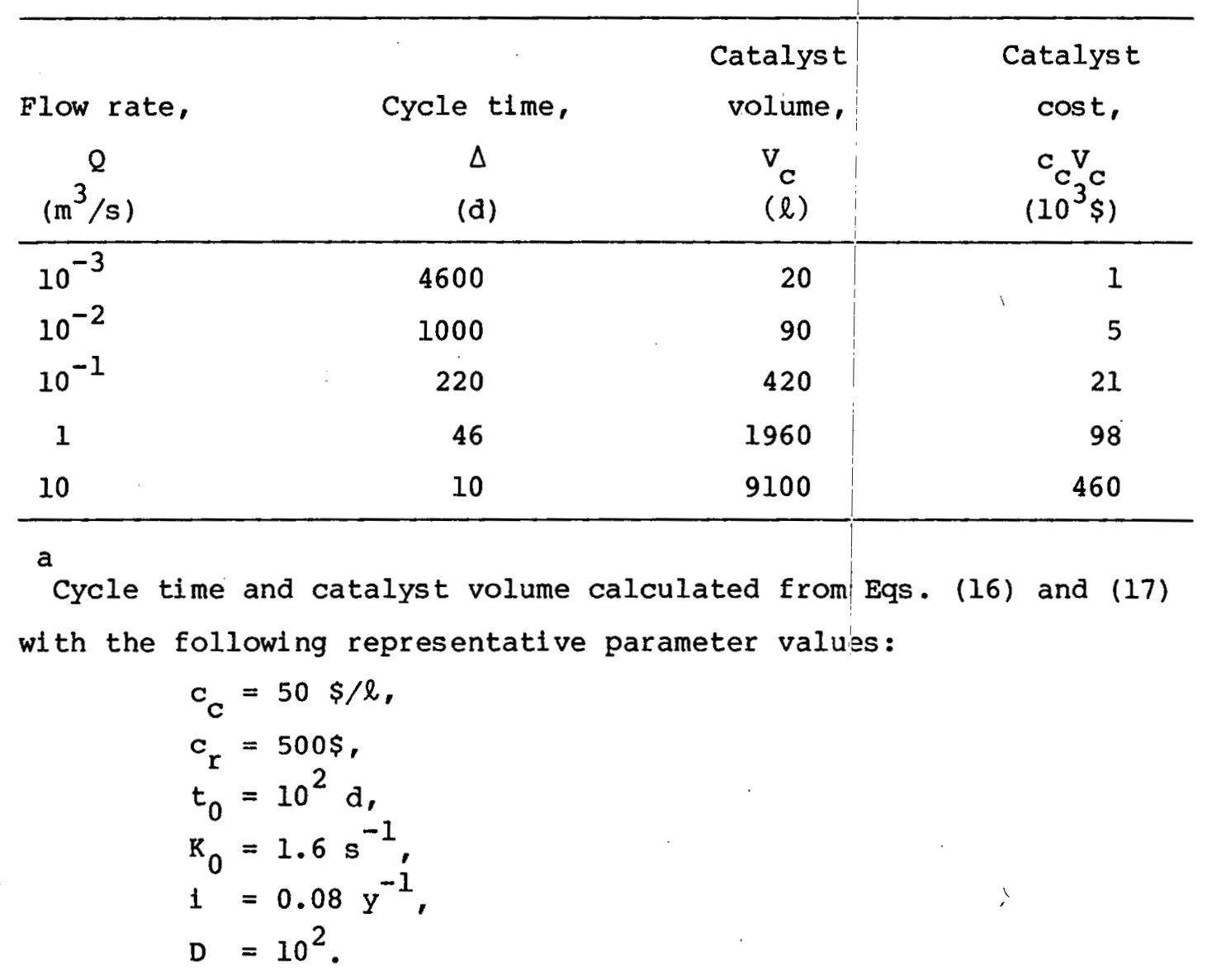




\section{SUMMARY}

We have characterized three precious-metal catalysts and measured kinetic rate coefficients for tritium/air oxidation at ambient temperature. Our results represent a significant expansion of the documented rate data available in the literature. Kinetic data are essential for the design of catalytic converters used in tritium scrubbing systems.

Our experiments were carried out with an apparatus that was originally developed for the maintenance of tritium-contaminated equipment. This unique apparatus consists of a Lucite enclosure with a large access door, and includes the capability of processing the enclosure atmosphere by recirculation through a cleanup system. Concentration levels of tritium gas and tritiated water vapor are measured and discriminated by means of dual-ionization chambers with an intermediate adsorption bed.

During the course of our investigations we observed the buildup of tritiated methane in our tritium-source vessel in agreement with a previous investigator. We observed that ambient-temperature catalysis is ineffective in promoting methane oxidation, a finding consistent with earlier studies.

Each of the dispersed-metal catalysts is extremely effective in promoting tritium oxidation in comparison with self-catalyzed atmospheric conversion; equivalent first-order rate constants are higher by roughly 9 orders of magnitude. Electron-microprobe scans reveal that the dispersed metal is deposited near the outer surface of the catalyst pellet, with metal concentration decreasing exponentially from the pellet surface. The platinum-based catalyst is more effective than the palladium catalysts on a surface-area basis by about a factor of 3 .

Additional conclusions that apply to all three catalysts may be summarized as follows:

- The catalytic reaction in air is first-order in tritium concentration in the range $10-10^{5} \mu \mathrm{Ci} / \mathrm{m}^{3}$ (4 ppt-40 ppb).

- Addition of a hydrogen carrier gas to the tritium-contaminated air flow is unnecessary.

- Catalytic activity in air is independent of moisture level in the range 100-4000 ppm.

- Catalysts should be activated before use with a hot hydrogen treatment. 
- Catalytic activity declines with time of exposure to alr after activation. The decline roughly follows a power-law decay with an exponent of $-1 / 2$.

- In situ reactivation with 18 hydrogen is ineffective. Reactivation in a heated, 1008 hydrogen environment effectively restores initial catalytic activity.

- A model for optimization of the catalyst reactivation cycle is proposed. Based on this model, we find that optimum catalyst volume scales with flow rate to the $2 / 3$ power.

- Ambient-temperature tritium oxidation is cost-effective for small- to intermediate-scale cleanup systems. A heateci catalyst is desirable for large-scale systems. 
1. S. O. Dean, The United States Magnetic Fusion Energy Program, U.S. Department of Energy, DOE/ET-0072 (1978), pp. 103-104.

2. J. L. Anderson and R. H. Sherman, Tritium Systems Test Assembly, Los Alamos Scientific Laboratory, Proposal LA-6855-P (1977).

3. K. E. Lind et al., "Tritium Handling Sys tem for TFTR and PITR," in Proc. 3rd Topical Meeting on the Technology of Controlled Nuclear Fusion, U.S. DOE, CONF-780508 (1978).

4. M. F. Singleton and R. M. Allre, "Traps for Scavenging Hydrogen Isotopes," in ibid.

5. C. L. Folkers and R. J. Cena, Tritium-Containment Systems: A Tradeoff Study, Lawrence Livermore Laboratory, Livermore, Calif., UCRL-52627 (1978).

6. A. E. Sherwood, "Tritium Removal from Alr Streams by Catalytic Oxidation and Water Adsorption," in Proc. 24th Conf. Remote Systems Tech. CRSTBJ 24, 1976 (American Nuclear Society, Hinsdale, Ill., 1976).

7. J. C. Bixel and C. J. Kershner, "A Study of Catalytic Oxidation and Oxide Adsorption for the Removal of Tritium from Air," Proc. 2nd

Envir. Prot. Conf., U.S. AEC, WASH-1332 (1974).

8. H. G. Ostlund and A. S. Mason, "Atmospheric HT and HTO I. Experimental Procedures and Tropospheric Data 1968-1972," Tellus 26, 91 (1974).

9. J. Wei, "The Catalytic Muffler," In Chemical Reaction Engineering Revi ews, Adv. Chem. Serles 148 (Amer. Chem. Soc., Wash., D.C., 1975).

10. J. R. Anderson, Structure of Metallic Catalysts (Academic Press, New York, 1975), pp. 190-202.

11. A. E. Sherwood, "Dynamic Model of Tritlum Cleanup in an Enclosure with Wall Diffusion," Proc. 3rd Topical Meeting on the Technology of Controlled Nuclear Fusion, op. cit.

12. G. A. Morris, Methane Formation in Tritium Gas Exposed to Stainless Steel, Lawrence Livermore Laboratory, Livermore, Calif., UCRL-52262 (1977).

13. D. J. Bender (ed.), Reference Design for the Standard Mirror Hybrid Reactor, A Joint Report by Lawrence Livermore Laboratory and General Atomt c Company, UCRL-52478, GA-A14796 (1978), p. 236. 
14. F. A. Kulacki and D. Gidaspow, "Convective Diffusion in a Parallel Plate Duct with One Catalytic Wall-Laminar Flow-First order Reaction Part II: Experimental," Can. J. Chem. Eng. 45, 72 (1967).

15. J. D. Eakins and W. P. Hutchinson, "The Radiolocilcal Hazard from the Conversion of Tritium to Tritiated Water in Air by Metal Catalysts," in Tritium, A. A. Moghissi and M. W. Carter (eds.), Messenger Graphics, Phoenix, Arizona (1971).

16. A. Ozaki, Isotoplc Studies of Heterogeneous Catalysis (Academic Press, New York, 1977), p. 203.

17. L. F. Belovodskil et al,, "Oxidation of Tritium in Air Under the Action of Intrinsic Radiation," Atomic Energy (translation of Atomnaya Energiya), 38, 488 (1975). 